\title{
USPIO-PEG Nanoparticles Functionalized with a Highly Specific Collagen-binding Peptide: a Step towards MRI Diagnosis of Fibrosis
}

\begin{tabular}{|c|c|}
\hline Journal: & Journal of Materials Chemistry $B$ \\
\hline Manuscript ID & TB-ART-04-2020-000887.R1 \\
\hline Article Type: & Paper \\
\hline $\begin{array}{r}\text { Date Submitted by the } \\
\text { Author: }\end{array}$ & 19-May-2020 \\
\hline Complete List of Authors: & 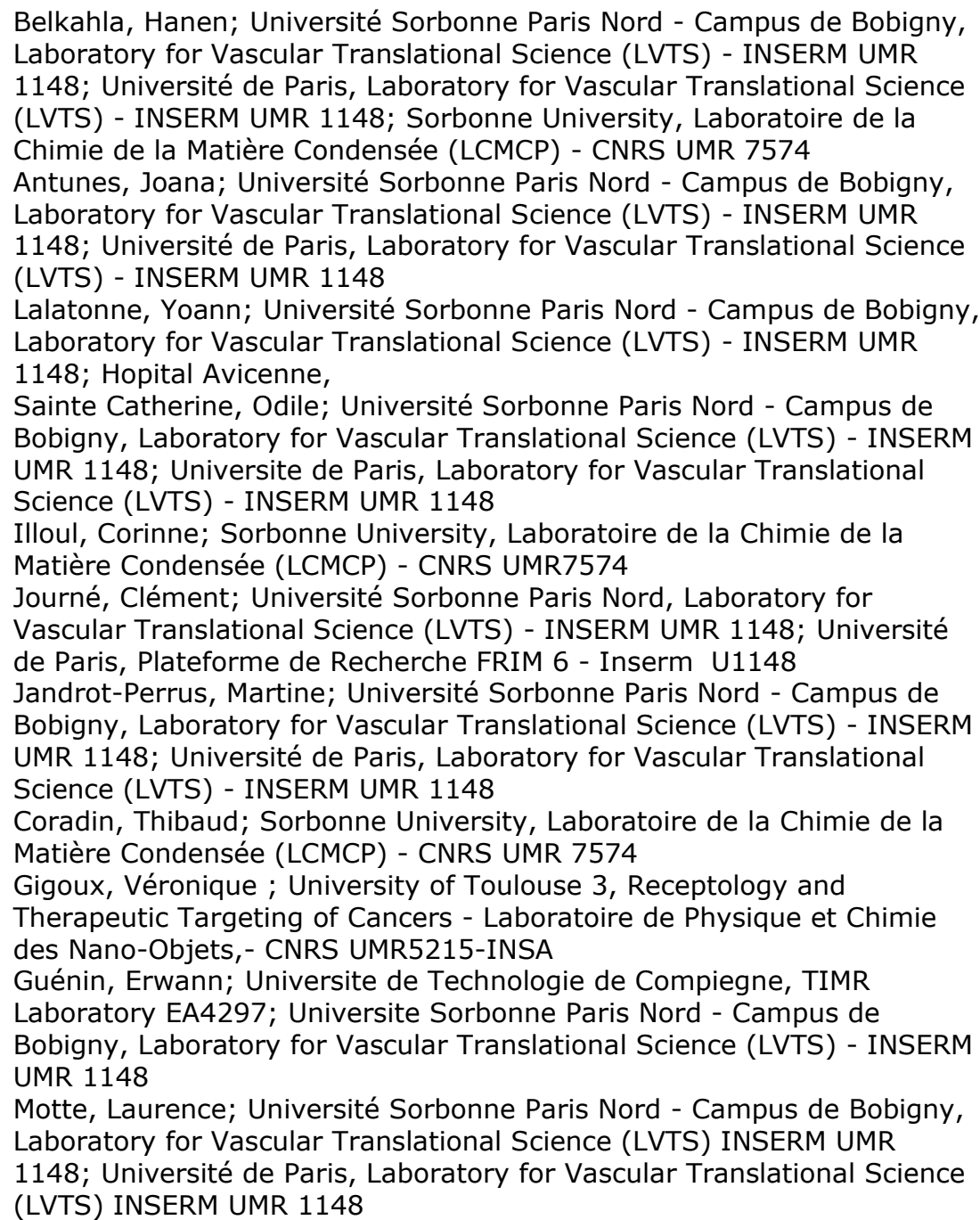 \\
\hline
\end{tabular}


Helary, Christophe; Sorbonne University, Laboratoire de la Chimie de la Matière Condensée (LCMCP) - CNRS UMR 7574

\section{SCHOLARONE ${ }^{m}$ Manuscripts}




\section{USPIO-PEG Nanoparticles Functionalized with a Highly Specific Collagen-binding Peptide: a Step towards MRI Diagnosis of Fibrosis}

Hanene Belkahla ${ }^{1,2 x}$, Joana C. Antunes ${ }^{1 x}$, Yoann Lalatonne ${ }^{1,3}$, Odile Sainte Catherine ${ }^{1}$, Corinne Illoul ${ }^{2}$, Clément Journé ${ }^{4}$, Martine Jandrot-Perrus ${ }^{1}$, Thibaud Coradin'2, Véronique Gigoux ${ }^{6}$, Erwann Guenin ${ }^{1,7}$, Laurence Motte ${ }^{1 *}$, Christophe Helary ${ }^{2 *}$

1- Université Sorbonne Paris Nord, Laboratory for Vascular Translational Science, LVTS, INSERM, UMR 1148, F- 93000 Bobigny, France.

Université de Paris, F-75018, Paris, France ; INSERM, F-75018, Paris, France

E-mail:laurence.motte@univ-paris13.fr

2-Sorbonne Université, CNRS, Laboratoire de la Chimie de la Matière Condensée (LCMCP), Paris, F-75005, France.

E-mail:christophe.helary@sorbonne-universite.fr

3- AP-HP, Hôpital Avicenne, Services de Biochimie et de Médecine Nucléaire service, F-93009 Bobigny, France

4- INSERM, UMR 1148, LVTS, Université de Paris, F-75018, Université Paris Nord, F-93430, Villetaneuse, France Inserm, Plateforme de Recherche FRIM 6-Inserm U1148, Université de Paris, Paris, France

5-INSERM ERL1226-Receptology and Therapeutic Targeting of Cancers, Laboratoire de Physique et Chimie des Nano-Objets, CNRS UMR5215-INSA, Université de Toulouse III, F31432 Toulouse, France 6-Sorbonne Universités, Université de Technologie de Compiègne, Integrated Transformations of Renewable Matter Laboratory (EA TIMR 4297 UTC-ESCOM), Compiègne, France

$X:$ These authors contributed equally

* Corresponding authors: Christophe Helary and Laurence Motte

Email: christophe.helary@sorbonne-universite.fr 


\section{Abstract:}

Fibrosis is characterized by a pathologic deposition of collagen I, leading to impaired function of organs. Tissue biopsy is the gold standard method for the diagnosis of fibrosis but this is an invasive procedure, subject to sampling errors. Several non-invasive techniques such as magnetic resonance imaging (MRI) using non-specific probes have been developed but they are not fully satisfying as they allow diagnosis at a late stage.

In this study, Collagelin, a collagen-binding peptide has been covalently linked using click chemistry to pegylated Ultra Small Super Paramagnetic Iron Oxide Nanoparticles (USPIO-POPEG-Collagelin NPS) with the aim of diagnosing fibrosis at an early stage by MRI. USPIO-POPEG-Collagelin NPs showed a high affinity for collagen I, two times higher than that of free Collagelin whereas not peptide labeled USPIO NPs (USPIO-PO-PEG-yne) did not present any affinity. NPs were not toxic for macrophages and fibroblasts. Diffusion through collagen hydrogels concentrated at 3 and $10 \mathrm{mg} \cdot \mathrm{mL}^{-1}$ revealed a large accumulation of USPIO-POPEG-Collagelin NPs within the collagen network after 72 hours, c.a 3 times larger than that of unlabeled USPIO, thereby evidencing the specific targeting of collagen I. Moreover, the quantity of USPIO-PO-PEG-Collagelin NPS accumulated within hydrogels was proportional to the collagen concentration. Subsequently, the NPs diffusion through collagen hydrogels was monitored by MRI. The MRI T2 time relaxation decreased much more significantly with depth for USPIO-PO-PEG-Collagelin NPs compared to unlabeled ones. Taken together, these results show that USPIO-PEG-Collagelin NPs are promising as effective MRI nanotracers for molecular imaging of fibrosis at an early stage.

Keywords: USPIO, Fibrosis, MRI Probe, Collagen I, Collagelin peptide, Collagen Hydrogels. 


\section{Introduction}

Fibrosis is the outcome of most chronic diseases and represents an increasing burden for public health. Nearly $45 \%$ of all deaths are associated with chronic fibro-proliferative diseases, the primary characteristic of which is the adverse remodeling of the extracellular matrix (ECM) ${ }^{1,2}$. Fibrosis following physical, metabolic, ischemic, infectious, inflammatory, or immunological injury can occur in any tissue and organ, and cause destruction of the affected structures such as lung, kidney, heart and liver ${ }^{3,4}$. Fibrosis is characterized by an extensive and pathological deposition of type I collagen (collagen I) produced by myofibroblasts, leading to impaired function of organs. The activation and the differentiation of fibroblasts into myofibroblasts is another important characteristic of the disease 5 . Tissue biopsy is the gold standard method for the diagnosis of fibrosis but this is an invasive procedure, subject to sampling errors and inter-observer variability ${ }^{6}$. Several non-invasive techniques (NITs) have been developed in order to delineate and quantify fibrosis for an early diagnosis. Among them, ultrasonography (US), single photon emission computed tomography (SPECT), positron emission tomography (PET) and magnetic resonance imaging (MRI) are the most studied ${ }^{1,2,7 .}$ Gadolinium-based contrast agents are often used in MRI as they allow the quantification of ECM volume via T1 Mapping. Recently, Akam et al developed hydrazine-bearing gadolinium as T1 MRI contrast agents which had a high affinity for allysine residues and accumulated in fibrosing lung tissue ${ }^{8}$. However, these currents NITs are not fully satisfying as they generally reflect anatomical or diffusion/perfusion-related alterations in the affected organs, thus providing information in the late-stage organ fibrosis, which is already characterized by irreversible anatomic and hemodynamic impairments. Hence, a crucial need of fibrosis targeting exists to detect the pathology at an early stage by increasing the signal/noise ratio. Since collagen I is the main component of fibrotic tissue, its 
targeting is of wide interest for molecular imaging ${ }^{9}$. Several collagen-like peptides such as EP3533 and CNA 35, coupled with gadolinium, have been developed for molecular imaging 1, 10, 11. More recently, the small cyclic peptide named "Collagelin", has revealed particularly promising as a targeting agent because it mimics the ability of platelet glycoprotein VI (GPVI) to bind to type I and type III collagens and therefore behaves as a collagen-specific probe ${ }^{12}$, 13. Furthermore, potential of Collagelin as an effective probe has been confirmed by two independent studies ${ }^{14,15}$. Collagelin was demonstrated to be a sensor of fibrosis in vitro, ex vivo and in vivo after labeling by ${ }^{99 \mathrm{~m} T c}$. The development of the method for clinical use was however limited by an insufficient target-to-background signal.

Owing to their magnetic properties, Ultra Small Super Paramagnetic Iron Oxide Nanoparticles ,USPIO NPs (particles with a diameter less than $50 \mathrm{~nm}$ ), represent one of the most promising systems to elaborate MRI contrast agents ${ }^{16-18 . ~ U S P I O ~ N P s ~ p o s s e s s ~ h i g h e r ~}$ magnetic properties than gadolinium-based contrast agents ${ }^{19,} 20$. In addition, their ability to be coupled with a biomolecule which targets the pathological tissue is of great interest ${ }^{21-26 .}$ The tracer accumulates in or at the vicinity of the selected target leading to specific retention into tissues of interest. USPIO NPs are highly biocompatible due to the natural metabolism of iron in vivo. Indeed, several studies demonstrated that internalized USPIO NPs are biotransformed into poorly-magnetic iron species and degraded over weeks/months. Iron is further recycled into ferritin storage proteins ${ }^{27-29 .}$ In extracellular matrices, clustering of ligands is a common strategy to enhance the strength and specificity of their biological interactions with cells ${ }^{30}$. On this basis, we propose to develop novel MRI contrast agents for heart fibrosis detection based on the use of USPIO NPs decorated with Collagelin, where the confinement of peptides on the NP surface should result in their clustering and therefore increase their collagen-binding efficiency and fibrosis 
targeting. Here, we report the design of such NPs and their MRI properties. In vitro assays were performed to assess their cytocompatibility on relevant cell lines (macrophages and fibroblasts) and their targeting ability, using 3D type I collagen-based models of fibrotic tissues at an early stage.

\section{Experimental Section}

\subsection{Materials}

Phosphonate-poly(ethylene glycol) PO-PEG-NH 2 (SP-1P-14-001, MW 2100 g.mol-1) was purchased from Specific Polymers (Castries, France). Heptynoic acid, methylene chloride and reagents for particle synthesis were bought at Sigma-Aldrich (Saint Louis, MO, USA), while 1(3-Dimethylaminopropyl)-3-ethylcarbodiimide hydrochloride (EDC) and Nhydroxysuccinimide were acquired from Alfa Aesar (Karlsruhe, Germany). Biotinylated Collagelin peptide was purchased from Proteogeomic (Schiltigheim, France). Nornal Human Dermal Fibroblasts were obtained from PromoCell. Dulbecco's modified Eagle culture medium (DMEM), Roswell Park Memorial Institute medium (RPMI1640), TrypsinEDTA, Fetal Bovine Serum (FBS), Streptomycin/Penicillin were purchased from Gibco-BRL

\subsection{Synthesis of PO-PEG-yne}

The PO-PEG-yne was synthesized using carbodiimide chemistry in a two-step procedure (activation and conjugation) at room temperature: $4 \mathrm{~h}$ for the activation step and overnight for the conjugation step (orbital shaking of $900 \mathrm{rpm}$ ) (see ESI, Figure ESI 1).

First, the carboxylic acid function of 6-heptynoic acid $(1.26 \mathrm{~g}, 10 \mathrm{mmol})$ was activated using 1-ethyl-3-(3-(dimethylamino)propyl)carbodiimide (EDC, $1.71 \mathrm{~g}, 11 \mathrm{mmol}, \mathrm{n}_{\mathrm{EDC}}=1.1 \mathrm{n}_{\mathrm{COOH}}$ ) and N-hydroxysuccinimide (NHS, $1.27 \mathrm{~g}, 11 \mathrm{mmol}, \mathrm{n}_{\mathrm{NHS}}=1,1 \mathrm{n}_{\mathrm{COOH}}$ ) in $50 \mathrm{~mL}$ 
dichloromethane. 
After evaporation of the solvent, the obtained yellow oil was dissolved in $50 \mathrm{~mL}$ diethyl ether and washed with water ( 3 times in $50 \mathrm{~mL}$ ). The ether phase was then evaporated so that the activated heptynoic ester product (white solid) could be collected (quantitative yield). The second step included the reaction of the amine functions of PO-PEG-NH $\mathrm{N}_{2}(2.1 \mathrm{~g}, 1 \mathrm{mmol})$ with the activated carboxylic acid functions. After adjusting the $\mathrm{pH}$ of the PO-PEG- $\mathrm{NH}_{2}$ water solution $(10 \mathrm{~mL})$ to 8 with sodium hydroxide $(\mathrm{NaOH}, 1 \mathrm{M}), \mathrm{N}, \mathrm{N}$-diisopropylethylamine (100 $\mu \mathrm{L}, 1,1 \mathrm{mmol}$ ) was added, and mixed overnight with NHS-ester activated heptynoic acid (290 $\mathrm{mg}, 1.3 \mathrm{mmol})$ dissolved in $(10 \mathrm{~mL})$ dimethylformamide $\left(\mathrm{DMF}, \mathrm{n}_{\text {ester }} / \mathrm{n}_{\mathrm{NH} 2 \text {-ester }}=1.3\right)$. The byproducts were extracted with diethyl ether and the PO-PEG-yne was kept in water phase and then lyophilized. The final compound was characterized by NMR and FTIR (Figure ESI 1).

\subsection{USPIO-PO-PEG-yne NP synthesis and surface functionalization}

Non-coated NPs were synthesized by the reaction of Iron (III) acetyl acetonate (1.1 mM) with of benzylalcohol $(10 \mathrm{~mL})$ at $250^{\circ} \mathrm{C}$ for 30 min under microwave irradiation on a Monowave 300 from Anton Paar (Anton-Paar, GmbH, Graz, Austria). The resulting suspension was separated using a neodymium magnet, and the precipitate was washed sequentially with dichloromethane followed by $\mathrm{NaOH}(1 \mathrm{M})$ and finally ethanol $90 \%$ (three times for each wash buffer). The NPs were re-dispersed in $\mathrm{pH} 2$ using $10^{-1} \mathrm{M}$ hydrochloric acid $(\mathrm{HCl})$ and subsequently washed three times by ultracentrifugation (Amicon 100 kDa, Merck Millipore). To coat the NPs with PO-PEG-yne, both compounds were mixed at $\mathrm{pH} 2$ with an equivalent mass (PO-PEG-yne/NP) ratio of 5 . Then, the excess of PO-PEG-yne coating molecules was removed through ultrafiltration (Amicon 100 kDa, Merck Millipore) and USPIO-PO-PEG-yne NPs were re-dispersed in water at $\mathrm{pH} 7$. 


\subsection{Collagelin coupling onto USPIO-PO-PEG-yne NPs}

The binding of biotinylated Collagelin to USPIO-PO-PEG-yne NPs was performed in a water/DMF (50/50) mixture in a one-step procedure under UV irradiation (360 nm). Briefly, the NPs in water at pH 7 and DMF was mixed with 1-hydroxycyclohexylphenyl ketone $(10 \%$ $\mathrm{v} / \mathrm{v}$ ) and the peptide with a molar ratio peptide/yne $=0.3$. The mixture was then placed under UV irradiation (4 hours). The modified particles were then ultracentrifuged several times with deionized water (Proteus X-spinner $100 \mathrm{kDa}$, Generon) and the $\mathrm{pH}$ was adjusted to 7 (Figure ESI 2).

\subsection{Physico-chemical characterizations}

Quantification of iron concentration was determined using a UV-visible spectrophotometer (UV-vis, V-630 UV-VIS Spectrophotometer, Jasco) based on a previously optimized colorimetric assay ${ }^{18}$. Additional measurements included a Multiple Collector - Inductively Coupled Plasma -Mass Spectrometry (MC-ICPMS, ThermoScientific, USA-Germany), and total reflection X-ray fluorescence spectroscopy (TXRF, S2 PICOFOX, Brucker). PO-PEG-yne coating and Collagelin grafting per particle were determined using the MC-ICPMS and TXRF, measuring simultaneously iron, phosphorous and sulfur content. For UV-vis and TXRF, samples were digested overnight in $\mathrm{HNO}_{3}(7 \mathrm{M})$ and $\mathrm{H}_{2} \mathrm{O}_{2}(20 \% \mathrm{v} / \mathrm{v})$ at $80^{\circ} \mathrm{C}$ and $500 \mathrm{rpm}$. For MC-ICPMS, samples were digested overnight in a $\mathrm{HNO}_{3} 65 \%$ at room temperature and then diluted in $\mathrm{HNO}_{3}(2 \%)$ for the elemental analyses. TXRF data was collected over $300 \mathrm{~s}$ with a molybdenum tube excitation source operating at $50 \mathrm{kV} / 700 \mu \mathrm{A}$. Elemental concentrations were calculated using gallium standard solution $(1 \mathrm{~g} / \mathrm{l}, \mathrm{Kraft} \mathrm{GmbH})$ for each sample through the PICOFOX ${ }^{\mathrm{TM}}$ software included in the equipment. 
The hydrodynamic size and $\zeta$ potential of the NPs $([\mathrm{Fe}]=1.5 \mathrm{mM})$ were investigated by dynamic and electrophoretic laser light scattering (DLS and ELS), using a Zetasizer Nano ZS (Red Badge) ZEN 3600 device (Malvern Panalytical, UK), equipped with a $4 \mathrm{~mW}$ HeNe laser beam with a wavelength of $633 \mathrm{~nm}$ and a scattering angle of $173^{\circ}$ (for size measurements) and $13^{\circ}$ (for $\zeta$ potential measurements). Size and $\zeta$ potential values were automatically calculated through the DTS Nano v.6.30 software, using the Stokes-Einstein equation and the Henry equation with the Smoluchowski approximation, respectively. The dispersion solution used was ultrapure water (DLS) and $1 \mathrm{mM} \mathrm{KCl} \mathrm{(ELS).} \mathrm{Folded} \mathrm{capillary} \mathrm{cells} \mathrm{(DLS;} \mathrm{Sigma)} \mathrm{with}$ gold-plated electrodes (Malvern) were used. Data were acquired at $37^{\circ} \mathrm{C}$, in triplicate. Transmission Electron Microscope (TEM) images were obtained using a FEI Tecnai 12 (80 kV; Philips) with a $1 \mathrm{~K} \times 1 \mathrm{~K}$ KeenView camera (OSiS). Samples were prepared by depositing a drop of NP suspension carbon-coated copper grids (EMS) placed on a filter paper. The median diameter was deduced from TEM data measurements, simulating the diameter distribution with a log-normal function 31.

\subsection{Magnetic studies}

A Vibrating Sample Magnetometer (VSM Quantum Design, Versalab) was used for magnetic characterization. VSM measured the magnetization by cycling the applied field from -30 to + $30 \mathrm{kOe}$ with a step rate of $100 \mathrm{Oe} \mathrm{s}^{-1}$ and from -1.5 to $+1.5 \mathrm{kOe}$ with a step rate of $10 \mathrm{Oe} \mathrm{s}^{-1}$ for high and low magnetic field investigation respectively. Measurements were performed on USPIO solutions at $[\mathrm{Fe}]=9.1 \mathrm{mM}$ (corresponding to $[\mathrm{NP}]=0.7 \mu \mathrm{M}$ and $\left[\mathrm{Fe}_{3} \mathrm{O}_{4}\right]=0.7 \mathrm{~g} \cdot \mathrm{L}^{-1}$ ) at $300 \mathrm{~K}$. The ZFC curve was obtained by first cooling the system in zero field from $300 \mathrm{~K}$ to 50 K. Next, an external magnetic field of 100 Oe was applied, and subsequently the magnetization was recorded with a gradual increase in temperature. The FC curve was 
measured by decreasing the temperature in the same applied field. MRI acquisitions were performed on a $7 \mathrm{~T}$ small animal MR scanner (Pharmascan, Bruker Bio- Spin GmbH, Rheinstetten, Germany).

Relaxometry measurements: in order to generate $\mathrm{T} 2$ weighted images at various concentration of NPs $([\mathrm{Fe}]=0.010$ to $0.15 \mathrm{mM})$ at $25^{\circ} \mathrm{C}$ in agarose gel, and thus calculate $\mathrm{T} 2$ maps, a multi-slice-multiecho sequence $(T E=11,33,55,77,99 \mathrm{~ms} ; 16$ echos; echo spacing = $11 \mathrm{~ms}$ and $\mathrm{TR}=2500 \mathrm{~ms}$ ) with a slice thickness of $1 \mathrm{~mm}$ was used to generate highresolution coronal images (Matrix: $192 \times 192$, Pixel size: $0.234 \times 0234 \mathrm{~mm}$ ). By graphing changes in relaxation rate $R_{2}\left(R_{2}=1 / T_{2}\right)$ at different concentration, the slope represents the relaxivity $r_{2}$.

The collagen affinity of USPIO-PO-PEG-yne NPs and USPIO-PO-PEG-Collagelin NPs was also evaluated measuring the $T 2$ relaxation time variation $(V)$ calculated as $V=100 \times\left(T_{x}-\right.$ $\mathrm{T}_{\text {control }} / \mathrm{T}_{\text {control }}$ where $T_{\text {control }}$ is the T2 relaxation time of collagen matrice without NPs and $T_{x}$ the $\mathrm{T} 2$ relaxation time of the sample with NPs at the distance $\mathrm{x}$ in the matrice. A TurboRARET2 (RAREmethod) sequence (TE $=9 \mathrm{~ms} ; \mathrm{TR}=3,694.281 \mathrm{~ms} ; \mathrm{NEX}=4$ and RARE factor $=8$ ) was used to generate T2 weighted images (Matrix: $256 \times 256$ ) with a slice thickness of $1 \mathrm{~mm}$.

\subsection{Collagen Affinity Measurement: USPIO-PO-PEG-Collagelin NPs}

Collagelin ( 0 to $100 \mu \mathrm{g} \cdot \mathrm{mL}^{-1}$ ), USPIO-PO-PEG-Collagelin (with concentration adapted to 0 to $100 \mu \mathrm{g} \cdot \mathrm{mL}^{-1}$ of peptide at the surface) and USPIO-PO-PEG-yne NPs (concentration adapted to the concentration range of USPIO-PO-PEG-Collagelin) were incubated on microtitration wells, coated with $1 \mu \mathrm{g}$ of type I collagen. Bound peptides were detected using HRP-coupled extravidin and the HRP substrate O-phenyl-dianisidine. 


\subsection{Cell Culture}

The Normal human dermal fibroblasts (NHDF, Promocell) and the murine macrophage cell line Raw 264.7 were grown respectively in Dulbecco's Modified Eagle Medium (DMEM, Gibco BRL) and Roswell Park Memorial Institute (RPMI1640, Gibco BRL) medium, respectively. Media were supplemented with $10 \%$ heat-inactivated FBS (Gibco BRL), penicillin (100 units. $\left.\mathrm{mL}^{-1}\right)$, streptomycin $\left(100 \mu \mathrm{g} \cdot \mathrm{mL}^{-1}\right.$, from Gibco BRL) and fungizone (0.25 $\left.\mu \mathrm{g} \cdot \mathrm{mL}^{-1}, \mathrm{Gibco} \mathrm{BRL}\right)$, at $37^{\circ} \mathrm{C}$ in $95 \%$ humidity and a $5 \% \mathrm{CO}_{2}$ atmosphere. At confluence, cells were removed from cultured flasks by treatment with $0.1 \%$ trypsin and $0.02 \%$ EDTA (Gibco BRL). Cells were rinsed and resuspended in the supplemented DMEM or RPMI1640 medium. Of note, NHDF cells were used at passage 6-7 for the experiments.

\subsection{Fabrication of Collagen Hydrogels}

Collagen I was extracted from rat tail tendons, purified and adjusted to a concentration of 5 $\mathrm{mg} \cdot \mathrm{mL}^{-1}$ in $0.5 \mathrm{M}$ acetic acid as previously described ${ }^{32,}{ }^{33 .}$ Collagen purity was monitored by SDS-PAGE and concentration measured by hydroxyprolin titration ${ }^{34}$. Then, the collagen stock solution was diluted with acetic acid $(0.5 \mathrm{M})$ to obtain a $3 \mathrm{mg} \cdot \mathrm{mL}^{-1}$ solution or concentrated up to $10 \mathrm{mg} \cdot \mathrm{mL}^{-1}$ by slow evaporation in a safety cabinet. To prepare collagen hydrogels, $2 \mathrm{~mL}$ of solutions concentrated at 3 or $10 \mathrm{mg} \cdot \mathrm{mL}^{-1}$ were cast into cell culture inserts (pore size $8 \mu \mathrm{m}$ ) adapted to 6 well culture plates. Hydrogels were formed by placing samples under concentrated ammonia vapors for $24 \mathrm{~h}$. Last, collagen hydrogels were rinsed with sterile PBS for 3 days until the $\mathrm{pH}$ was stabilized at 7.4. Composite alginate/collagen hydrogels were fabricated using alginate beads and a collagen solution (10 mg. $\left.\mathrm{mL}^{-1}\right)$ in acetic acid $(0.5 \mathrm{M})$. Basically, alginate beads were formed by extrusion of a $1 \%$ solution through a $23-\mathrm{G}$ needle into a $\mathrm{CaCl}_{2}(1 \mathrm{M})$ solution under stirring. 
After 1 hour, beads were collected and rinsed 3 times in PBS. Then, collagen and alginate beads were mixed with the ratio $1: 1(\mathrm{v} / \mathrm{v})$. The mixture $(2 \mathrm{~mL})$ was poured into each transwell and gelled under ammonia vapors as described above.

Cellularized collagen hydrogels were prepared using a solution at $10 \mathrm{mg} \cdot \mathrm{mL}^{-1}$ in $17 \mathrm{mM}$ acetic acid ${ }^{35}$. Collagen solution $(600 \mu \mathrm{L})$ was mixed with culture medium $(1.1 \mathrm{~mL}), \mathrm{NaOH}$ $(100 \mu \mathrm{L}, 0.1 \mathrm{M})$ and a cell suspension $\left(200 \mu \mathrm{L}\right.$ at $1.0 \times 10^{6}$ cells. $\left.\mathrm{mL}^{-1}\right)$. The mixtures were poured into cell culture inserts (pore size $8 \mu \mathrm{m}$ ) adapted to 6 well culture plates and set in an incubator at $37{ }^{\circ} \mathrm{C}$ with $95 \%$ humidity and a $5 \% \mathrm{CO}_{2}$ atmosphere for $24 \mathrm{~h}$ before starting the diffusion experiment. The final collagen concentration in cellularized hydrogels was $3 \mathrm{mg} \cdot \mathrm{mL}^{-}$ 1.

\subsection{Diffusion experiments}

Transwells were cast into 6 well plates containing PBS $(2 \mathrm{~mL})$ in each well, liquid used as receptor compartment for particles. Then, $2 \mathrm{~mL}$ of USPIO-PO-PEG-yne NPs or USPIO-PO-PEGCollagellin NPs ( $C_{0}=100 \mu \mathrm{g} . \mathrm{mL}^{-1}$ in iron) were deposited onto the surface of hydrogels (Figure ESI 3). At selected time points, from 1 to 72 h, 3 samples were studied for each condition. For this purpose, supernatants (upper compartment), hydrogels and liquids in the lower compartment (reservoir) were collected and the iron content determined in each compartment using MC-ICPMS.

When cellularized hydrogels were used, PBS was replaced by DMEM complete medium and the samples incubated at $37^{\circ} \mathrm{C}$ with $95 \%$ humidity and a $5 \% \mathrm{CO}_{2}$ atmosphere during the time course of the experiment. At each time point, cellularized collagen hydrogels were digested collagenase $\left(30 \mathrm{U} \cdot \mathrm{mL}^{-1}\right)$ for $30 \mathrm{~min}$ at $37^{\circ} \mathrm{C}$ prior to analysis. Fibroblasts were collected after collagen hydrogel digestion by centrifugation. The iron content was measured in the 
supernatant (digested collagen) to determine the quantity of USPIO within the collagen network. Recovered cells were hydrolyzed by adding ultrapure distilled water and used for Fe quantification to determine the NP quantity within cells.

\subsection{NP cytocompatibility on cell culture in 2D and 3D collagen hydrogel}

The USPIO-PO-PEG-yne NPs and USPIO-PO-PEG-Collagelin NPs cytotoxicity was evaluated using murine macrophage cell line Raw 264.7 and Normal Human Dermal Fibroblasts (NHDF). Cell metabolic activity was evaluated using the MTT tetrazolium assay based on the ability of mitochondrial enzymes to reduce 3-(4,5-dimethylthiazol-2-yl)-2,5diphenyltetrazolium bromide (MTT) (Sigma, St Louis, Mo) into purple formazan crystals. cells were seeded in 96-well flat-bottom plates $\left(1 \times 10^{4}\right.$ per well) and grown overnight in supplemented DMEM or RPMI1640 medium containing 10\% FBS. Then, the medium was removed and cells were treated with increased concentrations of NPs ( 0 to $2000 \mu \mathrm{M} \mathrm{Fe}$ ) in supplemented DMEM or RPMI 1640 medium containing FBS ( $0.5 \% \mathrm{v} / \mathrm{v})$ for $24 \mathrm{~h}$. Ten microliter of MTT solution ( $\left.5 \mathrm{mg} \cdot \mathrm{mL}^{-1}\right)$ was added to each well and incubated for $2 \mathrm{~h}$ at $37^{\circ} \mathrm{C}$. After incubation, the cell culture medium was removed and dimethylsulfoxide (100 $\mu \mathrm{L})$ of were added to each well and incubate for $1 \mathrm{~h}$ at $37^{\circ} \mathrm{C}$ to dissolve the formed formalin crystals. After incubation, the absorbance (OD) values were measured at $570 \mathrm{~nm}$, using a Labsystems Multiskan MS microplate reader. Cell metabolic activity was calculated as a percentage from the control corresponding to cells devoided of NPs.

Cell metabolic activity of entrapped cells in collagen matrix was also determined through MTT assay. However, a solution of MTT in PBS $\left(5 \mathrm{mg} \cdot \mathrm{mL}^{-1}\right)$ was prepared and added to the immobilized cells. 


\subsection{Histology and Prussian blue staining}

After 72 hours in contact with USPIO NPs, pure or composite collagen hydrogels were fixed with PFA $(4 \% \mathrm{w} / \mathrm{v})$, then dehydrated with ethanol, butanol and embedded in paraffin. Five micrometer transverse sections were performed using a manual microtome (Stiassnie, France). Sections were stained with Prussian blue 5\% potassium ferrocyanide in water (Sigma) for $15 \mathrm{~min}$, then with Prussian blue 5\% potassium ferrocyanide in hydrochloric acid ( $5 \% \mathrm{w} / \mathrm{v})$ (Sigma-Aldrich) for $15 \mathrm{~min}$ and counterstained with hematoxyline (Sigma) for 5 min. Then, samples were rinsed with $\mathrm{dH}_{2} \mathrm{O}$ and dehydrated again using ethanol and toluene. Last, sections were mounted between glass and coverslip using the Eukitt mounting medium. Samples were observed at magnification X600 with a Nikon Eclipse E600 POL and pictures taken with a Nikon DS-Ri1 camera

\subsection{Statistical analysis}

Results are presented as mean \pm standard deviation. Statistical analysis was performed using GraphPad Prism (version: 5.0a). Statistical significance was assessed using Mann-Whitney statistical test. The level of significance in all statistical analyses was set at a probability of $p$ $<0.05$.

\section{Results and Discussion}

\subsection{USPIO-PO-PEG-Collagelin NP synthesis and physico-chemical characterization}

USPIO NPs with an average diameter of $8.6 \pm 1.3 \mathrm{~nm}$ and a hydrodynamic diameter of $24.5 \pm 12.0 \mathrm{~nm}$ (Figure 1A) were synthesized using microwave non-aqueous sol-gel synthesis in a two-step process, following a previously-described procedure ${ }^{17,24}$ The NPs were 
surface-passivated with phosphonate polyethylene glycol (PEG) $\left(M_{W}=2100 \mathrm{~g} \mathrm{~mol}^{-1}\right)$ bearing an alkyne group as terminal functionality (PO-PEG-yne) (Figure ESI 2).

Transmission Electronic Microscopy (TEM) images showed well-dispersed USPIO-PO-PEG-yne NPs with spherical morphology and a narrow size distribution (Figure 1B). The organic layer ${ }^{36}$ of about $0.9 \mathrm{~nm}$ around the NPs (Figure 1B) was attributed to the PEG coating. Considering that nude nanoparticles had a zero charge potential at $\mathrm{pH}=7.4$, the negative zeta potential equal to $-24.3 \pm 1.7 \mathrm{mV}$, measured after surface functionalization, confirmed the efficient binding of the PO-PEG-yne to the NP surface. In addition, it could be expected a monodentate binding to USPIO surface, knowing that the phosphonic acid function possesses two pKa values, $\mathrm{pKa}=2.7$ and $\mathrm{pKa}=7.8^{37}$. The number of PO-PEG-yne molecules per particle determined by MC-ICPMS and TXRF analysis corresponded to an average of $312 \pm 58$ and $370 \pm 27$ PO-PEG-yne /NP, respectively.

The alkyne functions at the outer surface of the nanoparticle were used for the covalent binding of the biotinylated Collagelin using a thiol-yne reaction ${ }^{38}$. This water-compatible photochemical reaction occurred under UV irradiation. The peptide was used in its cyclic form, as the thiol-yne reaction is a radical reaction the disulphide bond was broken in an homolytic manner in the reaction conditions and the sulphide radicals reacted on the triple bond (Figure ESI 2). This was confirmed by doing the reaction in two steps: first reduction of the disulphide bond and then coupling of the peptide with thiol function to the USPIO-POPEG-yne NPs. The result in this latter case was completely identical in terms of grafted peptide quantity and affinity.Compared to USPIO-PO-PEG-yne NPs, the hydrodynamic diameter of USPIO-PO-PEG-Collagelin NPs did not change whereas the zeta potential increase (Figure 1C). Noticeably, after one month, no drastic change in hydrodynamic diameter of the two nanoplatforms was measured (Table ESI. 1). The average number of 
Collagelin per NP was evaluated quantitatively by ICP and TXRF analysis, measuring sulfur content, since sulfur is part of the peptide chemical structure. We found an average of $18 \pm 2$ or $27 \pm 1$ peptide/NP corresponding to a thiol yne reaction yield of $6 \%$ and $7 \%$ using ICP-MS and TXRF values respectively. The thiol yne reaction yield is low but this could be related to the use of a cyclic peptide which induces a steric hindrance at the surface of the nanoparticles. For the other experiments we used an average value equal to 22 peptides/NP.
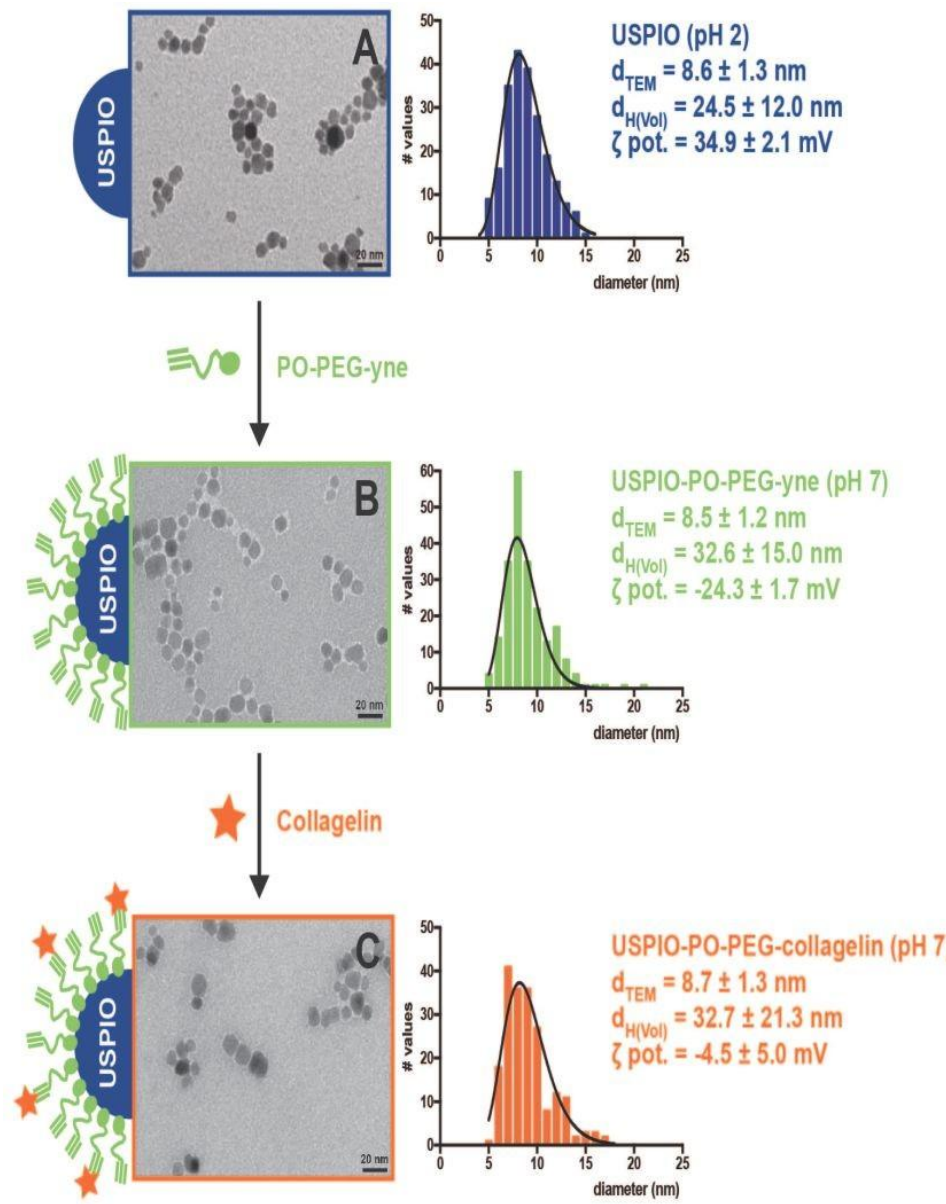
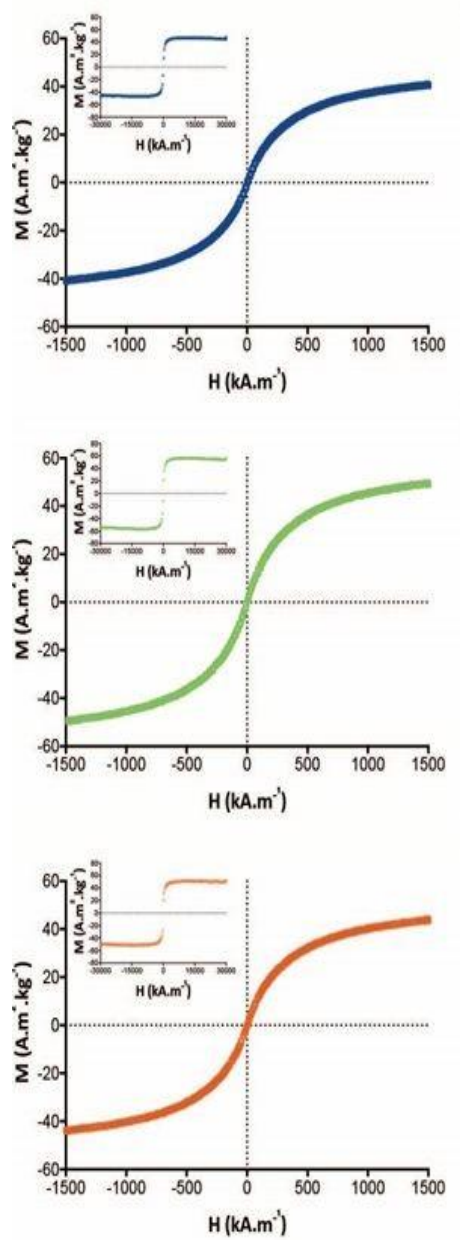

D

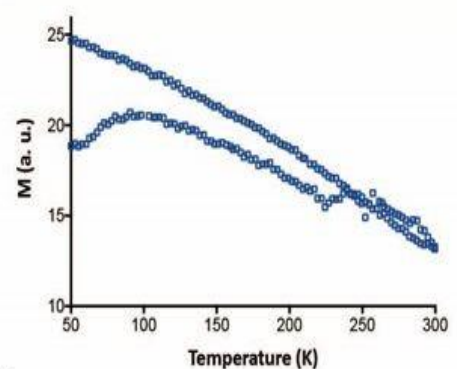

E

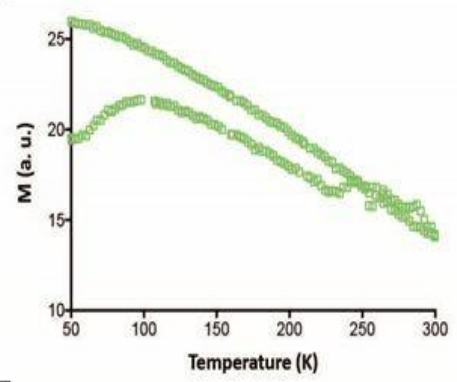

$\mathbf{F}$

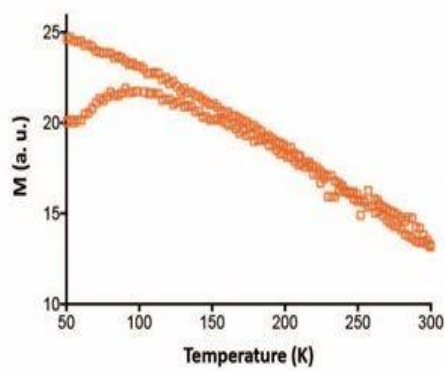

Figure 1: USPIO-PO-PEG-Collagelin NPs physico-chemical and magnetic characterization at each step of their synthesis. (A-C): TEM size distribution, TEM image, average crystalline diameter $\left(d_{\text {TEM }}\right)$, hydrodynamic diameter $\left(d_{H}\right)$, zeta potential $\left(\zeta_{\text {pot }}\right)$ value for bare USPIO NPS (A), USPIO-PO-PEG-yne NPs (B) and USPIO-PO-PEG-Collagelin NPs(C). (D-F): Magnetic 
characterization: low and high (insert) magnetization curves at room temperature (left graphs); and ZFC/FC curve (right graphs).

Magnetic properties of the NPs were explored using VSM and MRI 7T experiments.

Magnetization curves (Figure 1D-1F, left graphs) indicate that all NPs exhibited a superparamagnetic behavior. After the PEG-coating process, the magnetization saturation was maintained constant before $\left(48 \mathrm{emu}^{\mathrm{g}} \mathrm{g}^{-1}\right)$ and after coating $\left(51 \mathrm{emu}^{\mathrm{g}} \mathrm{g}^{-1}\right)$ as well as after covalent coupling with Collagelin $\left(51 \mathrm{emu}^{-1}{ }^{-1}\right)$, demonstrating that the phosphonate chelation did not alterate the NPs crystalline structure,.. The blocking temperature (Figure $1 \mathrm{D}-1 \mathrm{~F}$, right graphs) is found equal to $98 \mathrm{~K}$ for all samples, demonstrating that NPs interactions remains unchanged during the chelating and coupling processes. Transverse relaxivities $(\mathrm{r} 2)$ measured at 7T, with various concentration of NPs ([Fe] $=0.010$ to $0.15 \mathrm{mM})$ at $25^{\circ} \mathrm{C}$ in agarose gel, were found equal to 140 and $147 \mathrm{mM}^{-1} \mathrm{~s}^{-1}$ for USPIO-PO-PEG-yne and USPIO-PO-PEG-collagelin NPs respectively, suggesting high T2 contrasting effect (Figure ESI.4).

\subsection{Affinity of USPIO-PO-PEG-Collagelin for collagen}

Binding of uncomplexed Collagelin (free form), USPIO-PO-PEG- Collagelin and USPIO-POPEG-yne NPs to immobilized collagen was measured (Figure 2A). USPIO-PO-PEG- Collagelin suspensions and Collagelin solution were adjusted on peptide concentration. USPIO-PO-PEGCollagelin and USPIO-PO-PEG-yne suspensions were adjusted on Fe content. While USPIOPO-PEG-yne NPs did not bind to collagen (OD $<0.05)$, free Collagelin bound in a dosedependent manner to immobilized collagen and in a concentration range $\left(20-100 \mu \mathrm{g} \cdot \mathrm{mL}^{-1}\right)$ consistent with previous results ${ }^{13}$. Interestingly, the binding of USPIO-PO-PEG-Collagelin NPs 
to collagen was increased when compared to free Collagelin with a two fold higher signal for the $20 \mu \mathrm{g} / \mathrm{mL}$ Collagelin concentration. Hence, the clustering of Collagelin in its conformation at the NP surface drastically increase its affinity towards collagen I. One must note that free Collagelin affinity for collagen is driven by its cyclic nature ${ }^{13}$. Thus it appears that the thiolyne reaction was able to ensure the effective cyclization of the peptide at the surface of the NPs (Figure ESI.2) allowing for its clustering and good affinity.
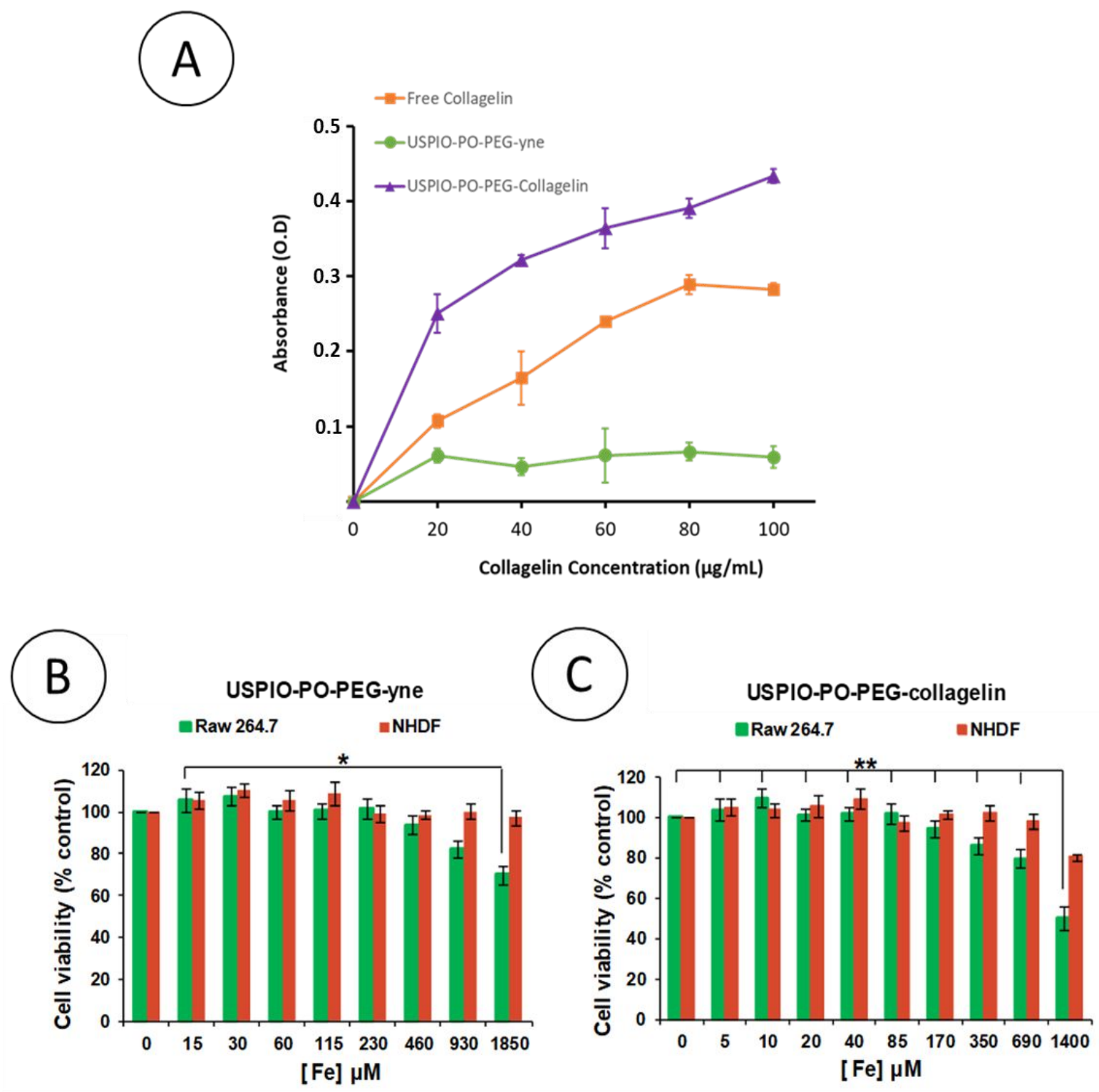
Figure 2: (A): Affinity of free Collagelin peptide, USPIO-Collagelin-yne and USPIO-PO-PEGCollagelin NPs for collagen I. (B,C): Cytocompatibility of USPIO-PO-PEG-yne (B) and USPIOPO-PEG-Collagelin NPs (C) towards Raw 264.7 macrophages and NHDF fibroblasts in basal static conditions, after incubation for $24 \mathrm{~h}$, with NPs at increasing concentration in iron. Results are shown as mean \pm SD from 5 independent experiments. Statistical significance was considered for $p<0.05\left({ }^{*}\right)$ or $p<0.001\left({ }^{* *}\right)$, using the Mann-Whitney test.

\subsection{Cytotoxicity of USPIO}

Cytotoxicity of the USPIO-PO-PEG-yne and USPIO-PO-PEG-Collagelin NPs were assessed in vitro using macrophages RAW 264.7 and Normal Human Dermal Fibroblasts (NHDF), (Figure 2B and C). No cytotoxicity was measured for fibroblasts over the whole investigated USPIO concentration range whereas cell viability decreased for macrophages at very high Fe concentration ( $>\sim 1000 \mu \mathrm{M}$ ). The tolerance towards USPIO of many cell types, including fibroblasts and hepatic cells is well-reported in the literature 20,39 , thereby explaining their large utilization in MRI. The cellular toxicity observed for macrophages at high Fe concentration may reflect their higher ability for NPs internalization due to their cellular function of debridement. Indeed, after internalization, USPIO were shown to be transferred and degraded in lysosomes, leading to reactive oxygen species production, lysosome damage and eventually to cell death 40 . Importantly, similar trends were obtained for USPIO-PO-PEG-yne and USPIO-PO-PEGCollagelin NPs, suggesting that the surface functionalization of the nanoparticles did not play a major role on their interactions with cells and further strengthening the idea that cytotoxicity for macrophages arises from NP accumulation following internalization rather than direct interactions with cell outer membrane. 


\subsection{Diffusion of USPIO-PO-PEG-yne and USPIO-PO-PEG-Collagelin through cellularized collagen hydrogels}

In a first step, collagen hydrogels at low concentration $\left(3 \mathrm{mg} \cdot \mathrm{mL}^{-1}\right)$ were selected and prepared in the presence of NHDFs to obtain tissue-like cellularized matrices. These hydrogels were used as a model of fibrotic tissue to simulate the beginning of this pathology when the collagen accumulation is not large. After the deposition of USPIO-PO-PEG-yne or USPIO-PO-PEG-Collagelin suspensions ( $100 \mu \mathrm{g} \cdot \mathrm{mL}^{-1}$ in Fe) on top of such constructs, the iron concentration was measured in each compartment (i.e. supernatant, hydrogel and reservoir) by MC-ICPMS at regular time points between $1 \mathrm{~h}$ and $72 \mathrm{~h}$ (Figure ESI 3). It is important to point out that USPIO NPs are stable in complete culture medium as shown by Plan Sangnier et al, so that it is possible to relate the iron concentration with the NP content ${ }^{41 .}$ After $1 \mathrm{~h}$, few particles had diffused through hydrogels regardless of the NPs type (Figure 3A). From 4 $h$, the concentration of iron decreased gradually in the upper compartment (supernatant) to reach ca. 60 and $40 \mu \mathrm{g} \cdot \mathrm{mL}^{-1}$ after 24 and $72 \mathrm{~h}$, respectively. The Fe concentrations for USPIOPO-PEG-Collagelin NPs measured in this compartment were similar to those of USPIO-POPEG-yne NPs at each time point. The Fe concentrations measured within collagen hydrogels slightly increased for the first $6 \mathrm{~h}$ of incubation but remained low $\left(5 \mu \mathrm{g} \cdot \mathrm{mL}^{-1}\right)$. It is important to remind that this concentration was measured on digested hydrogels after removal of cells. During this first period, both types of NPs had the same behavior of diffusion. In contrast, from $6 \mathrm{~h}$ to $72 \mathrm{~h}$, the concentration of Fe from USPIO-PO-PEG-Collagelin NPs measured within collagen hydrogels was significantly higher than that of USPIO-PO-PEG-yne NPs. At $24 \mathrm{~h}$, the concentration of Fe from USPIO-PO-PEG-Collagelin NPs was 1.5 times that 
of USPIO-PO-PEG-yne NPs reaching ca. $30 \mu \mathrm{g} \cdot \mathrm{mL}^{-1}$ after $72 \mathrm{~h}$ in incubation (Figure 3A). After $72 \mathrm{~h}$, the fraction of NPs entrapped within collagen hydrogels was $23 \%$ and $38 \%$ for USPIOPO-PEG-yne and USPIO-PO-PEG-Collagelin,NPs, respectively (Figure 3B). In addition, the iron concentrations from USPIO-PO-PEG-yne NPs measured in the reservoir was significantly higher than those of USPIO-PO-PEG-Collagelin NPs as soon as $4 \mathrm{~h}$ after the start of experiment. From $48 \mathrm{~h}$, the fraction of Fe in the reservoir was two-fold higher for USPIO-POPEG-yne compared to that for USPIO-Collagelin NPs (33\% vs 16\%).

The diffusion of USPIO NPs depends on the tortuosity of collagen hydrogels. The NPs have to go through the hydrogels, with sorption processes occurring between particles and the collagen fibrils ${ }^{42,43}$. Collagen hydrogels concentrated at $3 \mathrm{mg} \cdot \mathrm{mL}^{-1}$ exhibit a large porosity, with the pores diameter around 1-5 $\mu \mathrm{m}$, ie. 100 times larger than that of USPIO NPs ${ }^{35}$. This suggests a negligible impact of tortuosity on NPs diffusion. Regarding the sorption process, it was noticed that, at the end of the experiment the quantity of iron from USPIO-PO-PEGyne NPs was similar in each compartment, evidencing that an equilibrium of concentration has been established between the three parts of equal volume. This sign for a negligible sorption of USPIO-PO-PEG-yne NPs on collagen fibrils. It is in agreement with the well-known ability of PEG to prevent non-specific protein adsorption on (nano)-surfaces ${ }^{44}$. In contrast, the higher fraction of USPIO-PO-PEG-Collagelin NPs found within collagen hydrogels can be the affinity of Collagelin for collagen, favoring its sorption on protein walls of the porous network. Iron quantification was also performed within fibroblasts after collagen digestion by collagenase. The NPs uptake by fibroblasts was small (less than but $10 \mu \mathrm{g} \cdot \mathrm{mL}^{-1}$ ) but increased regularly with time up to $24 \mathrm{~h}$, with a lower absolute (Figure $3 \mathrm{~A}$ ) and relative (Figure 3B) content of Fe for USPIO-PO-PEG-Collagelin compared to USPIO-PO-PEG-yne NPs $(p<0.05)$. It 
is well-established that fibroblasts have the ability to internalize NPs but the internalization route and extent highly depends on NP size, charge/surface chemistry and state of aggregation ${ }^{45}$. Positively-charged NPs are usually internalized in a larger extent than neutral or negatively-charged ones, but this internalization is often associated with cell death ${ }^{46} \cdot \mathrm{In}$ our experiment, cell uptake was not associated to any toxicity (Figure ESI 5). Internalization of NPs, including USPIO, by cells also depends on their state of aggregation ${ }^{41}$. Thus, the difference in zeta potential value between the two types of NPs may, for some part, contribute to the observed variation in internalization efficiency. However, it must also be considered that because USPIO-PO-PEG-yne NPs interact less with the collagen network than USPIO-PO-PEG-Collagelin NPs, they should be more available to interact with cells. 
(A)
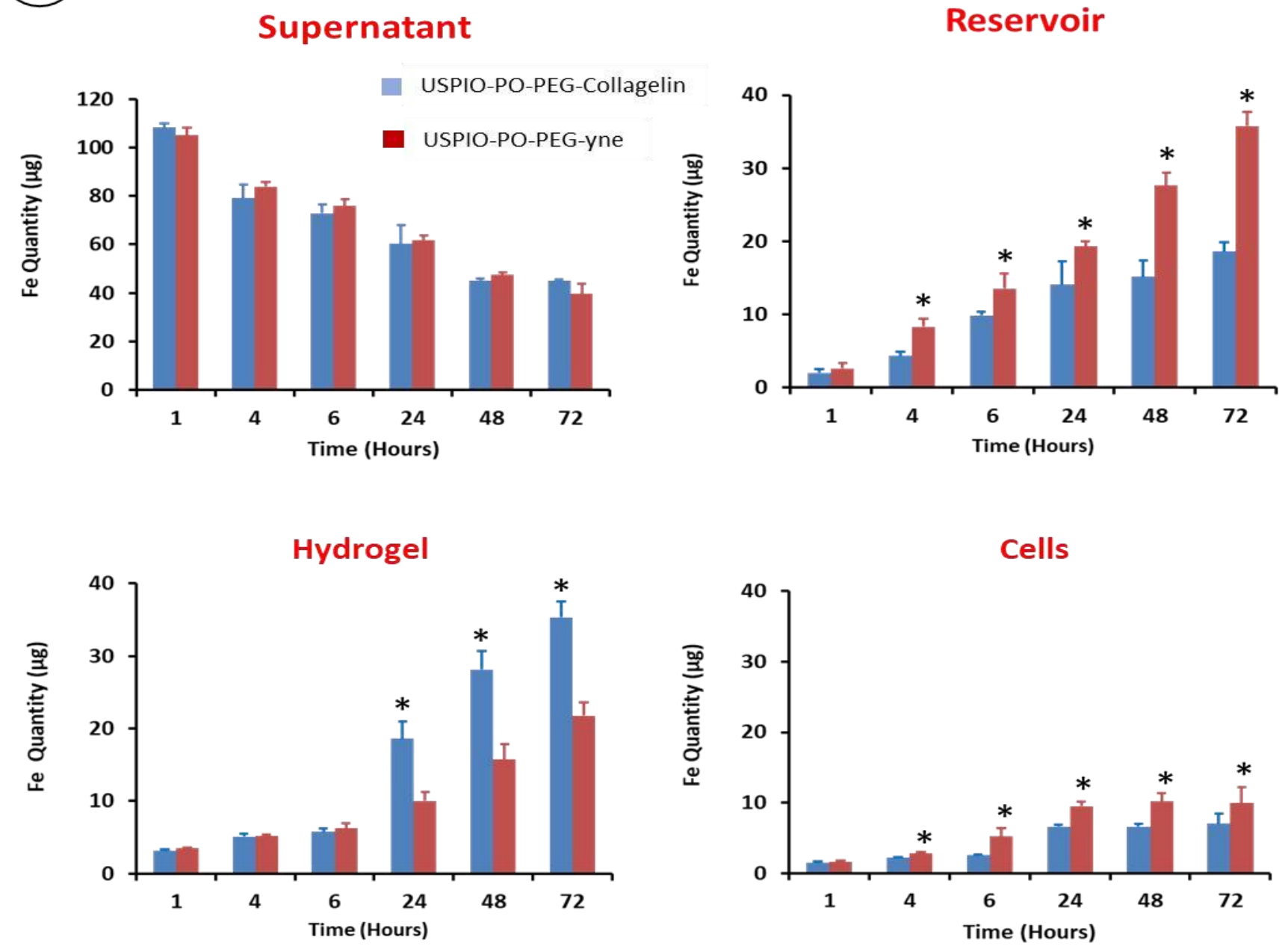

(B)
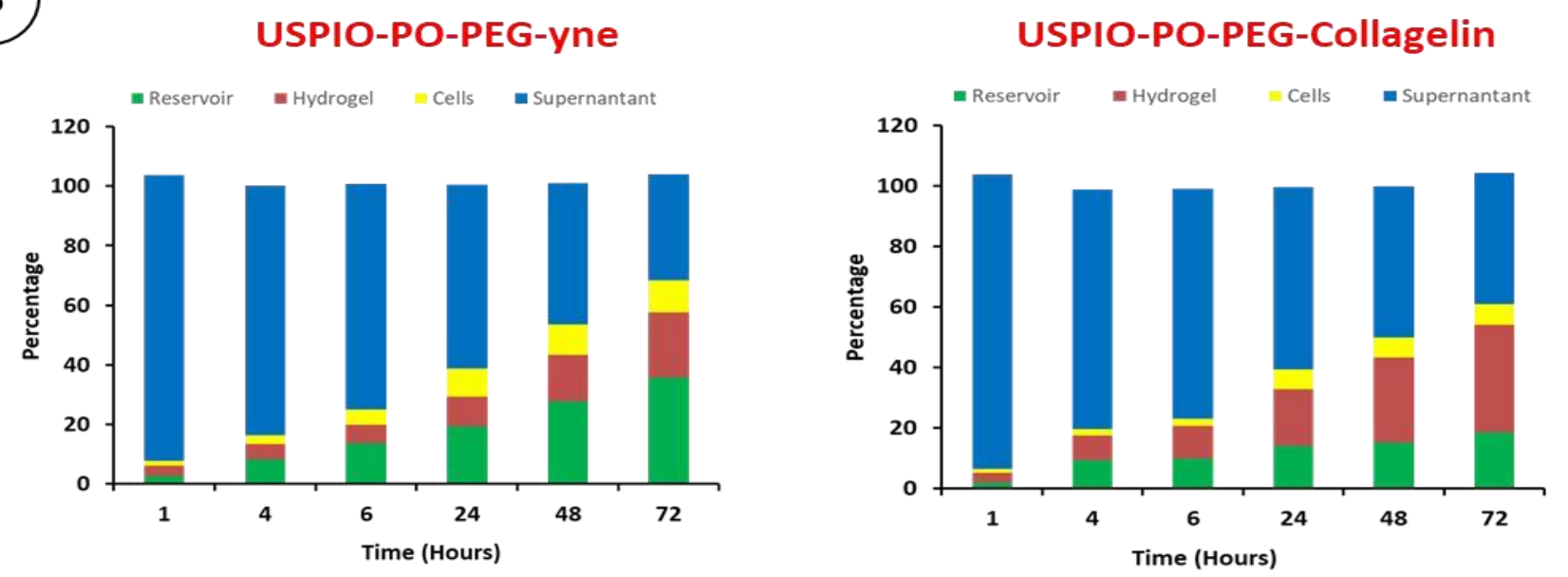

Figure 3: Diffusion and interaction of USPIO-PO-PEG-yne and USPIO-PO-PEG-Collagelin NPS within cellularized $3 \mathrm{mg} \cdot \mathrm{mL}^{-1}$ collagen hydrogels. $(*: p<0.05)$ using the Mann-Whitney test. 


\subsection{Interaction of USPIO-PO-PEG-yne and USPIO-PO-PEG-Collagelin with dense collagen hydrogels}

In order to study the effect of collagen network densification on NP retention, the previous experiments were reproduced in concentrated collagen hydrogels (10 mg.mL $\left.{ }^{-1}\right)$. Noticeably, the used protocol is not compatible with incorporation of cells prior gelation so that uncellularized matrices were studied. It is worth noticing that this collagen concentration is still much lower than that of mature fibrotic tissues (Collagen concentration more than 200 $\left.m g \cdot \mathrm{mL}^{-1}\right)$. The behavior of USPIO-PO-PEG-yne NPs diffusing through these gels was very similar to the less concentrated ones, with an equivalent repartition of the iron content in the three compartments after $72 \mathrm{~h}$ (Figure 4 and 5). One interesting observation is that the Fe content in the concentrated hydrogel is similar to the sum of hydrogel and cellular content in soft cellularized ones. This would mean that, in the absence of Collagelin, fibroblasts equally compete with collagen for interacting with NPs.

USPIO-PO-PEG-Collagelin NPs also had a similar general impact on NPs diffusion, with a systematic decrease in iron content in the reservoir and a significant increase $(p<0.05)$ in the hydrogel compared to USPIO-PO-PEG-yne NPs in the dense collagen gels (Figure 4A). However, the difference between the two types of NPs was much more marked than in the 3 $\mathrm{mg} \cdot \mathrm{mL}^{-1}$ constructs. This was especially clear after $72 \mathrm{~h}$ where the iron content within the hydrogel was 4 fold higher for USPIO-PO-PEG-Collagelin NPs compared to USPIO-PO-PEG-yne NPs (Figure 5B). As comparison, only a 1.5 fold higher USPIO-PO-PEG-Collagelin NPs compared to USPIO-PO-PEG-yne NPs was measured in the low concentrated gel after the same delay (Figure 4A). Such a higher retention of USPIO-PO-PEG-Collagelin NPs by the dense collagen gels almost equally impacted the content of the supernatant ( $8 \%$ for USPIOPO-PEG-Collagelin vs 35\% for USPIO-PO-PEG-yne) and of the reservoir (14\% for USPIO-PO- 
PEG-Collagelin vs. 34\% for USPIO-PO-PEG-yne NPs) (Figure 9). This suggests that the diffusion of NPs is still possible from the top to the bottom compartment, allowing to discard any pore blocking effect that could have sign for heterogeneous accumulation of the particles.

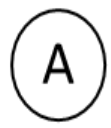

Supernatant
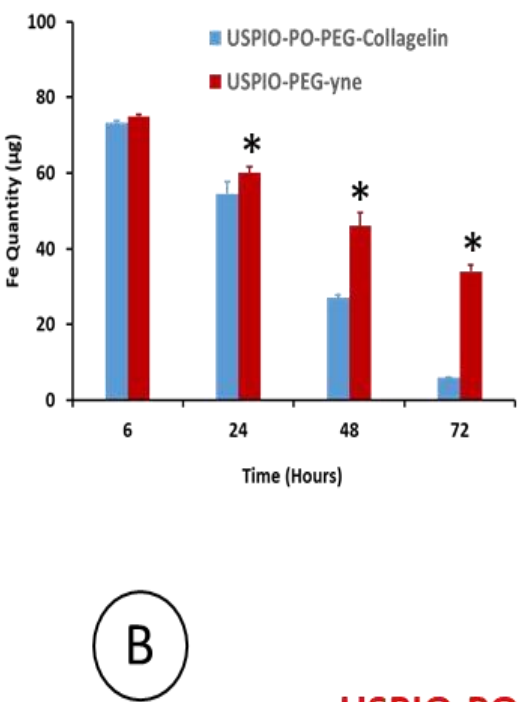

Hydrogel

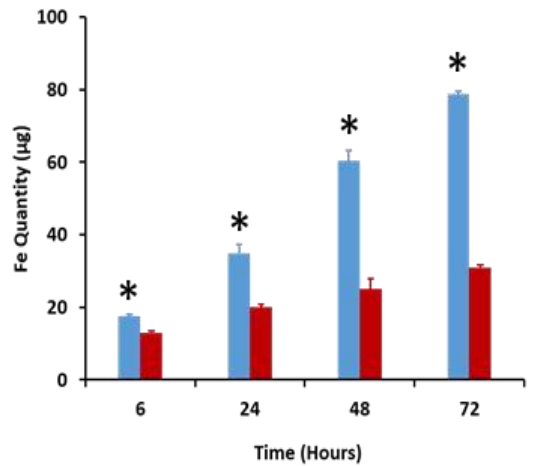

Reservoir

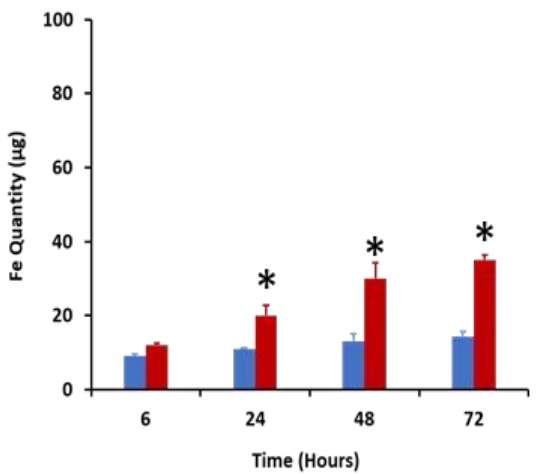

USPIO-PO-PEG-yne

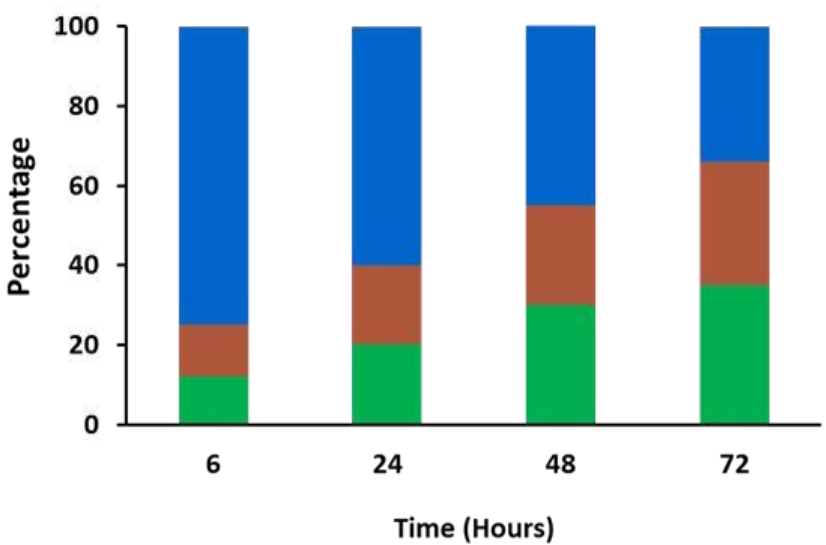

USPIO-PO-PEG-Collagelin

- Reservoir $\quad$ Hydrogel $\quad$ Supernantant

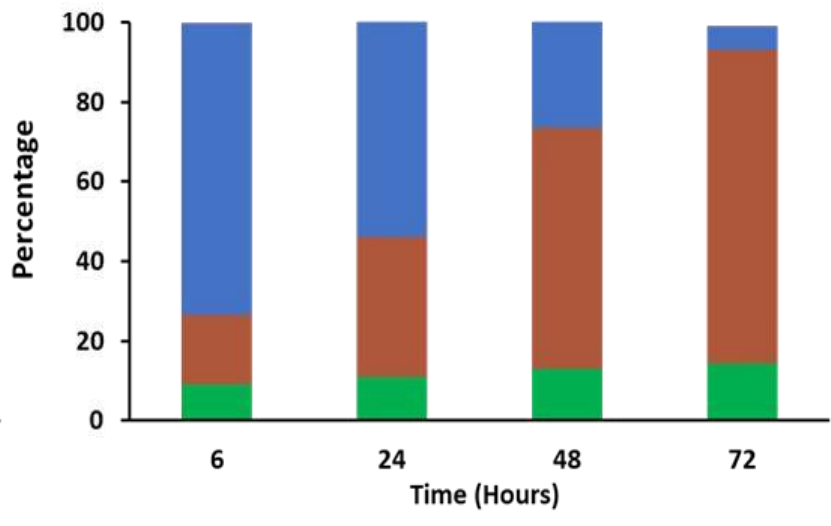

Figure 4: Diffusion and interaction of USPIO-PO-Peg-yne and USPIO-PO-PEG-Collagelin NPS within $10 \mathrm{mg} \cdot \mathrm{mL}^{-1}$ collagen hydrogels. $(*: \mathrm{p}<0.05)$ using the Mann-Whitney test. 
It has been previously shown that the kinetic diffusion of Si NPs through collagen hydrogels depended on the biopolymer concentration. The diffusion of $60 \mathrm{~nm}$ positively charged Si NP through $5 \mathrm{mg} \cdot \mathrm{mL}^{-1}$ hydrogels was much slower than that trough $1.5 \mathrm{mg} \cdot \mathrm{mL}^{-1}$ ones ${ }^{43}$. In addition, a lag phase appeared for the more concentrated gels. Here, the diffusion of $10 \mathrm{~nm}$ large USPIO-PO-PEG-yne NPs within $10 \mathrm{mg} \cdot \mathrm{mL}^{-1}$ hydrogels was similar to that within 3 $\mathrm{mg} \cdot \mathrm{mL}^{-1}$ ones without any lag phase, showing diffusion was not impacted by collagen concentration. This suggests that the modification of the collagen network porosity/tortuosity is not significant compared to the NP dimension. Therefore, the USPIOPO-PEG-Collagelin NPs must be mainly retained by the specific interaction of Collagelin ligands with type I collagen molecules. The amount of retained NPs should scale with the number of Collagelin targets, i.e. with the concentration of collagen. As fibrosis is characterized by the production and the accumulation of collagen I in tissues, USPIO-POPEG-Collagelin NPs could allow the early stage detection of fibrotic tissue ${ }^{1}$. The spatial distribution of NPs was analyzed on histological sections by staining iron using Prussian Blue (Figure 6). USPIO-PO-PEG-yne NPs were barely detectable within cellularized 3 $\mathrm{mg} \cdot \mathrm{mL}^{-1}$ hydrogels and were often located at the surface of dense collagen hydrogels, thereby evidencing the weak accumulation within collagen hydrogels. In contrast, USPIO-POPEG-Collagelin NPs diffused through the hydrogel thickness irrespective of the collagen concentration. It is worth noticing that the quantity of NPs observed in $10 \mathrm{mg} \cdot \mathrm{mL}^{-1}$ hydrogels was higher than that visible in $3 \mathrm{mg} \cdot \mathrm{mL}^{-1}$ ones (Figure 6). This shows that the accumulation of USPIO-PO-PEG-Collagelin NPs correlates with the hydrogel concentration, evidencing their high affinity for collagen. 


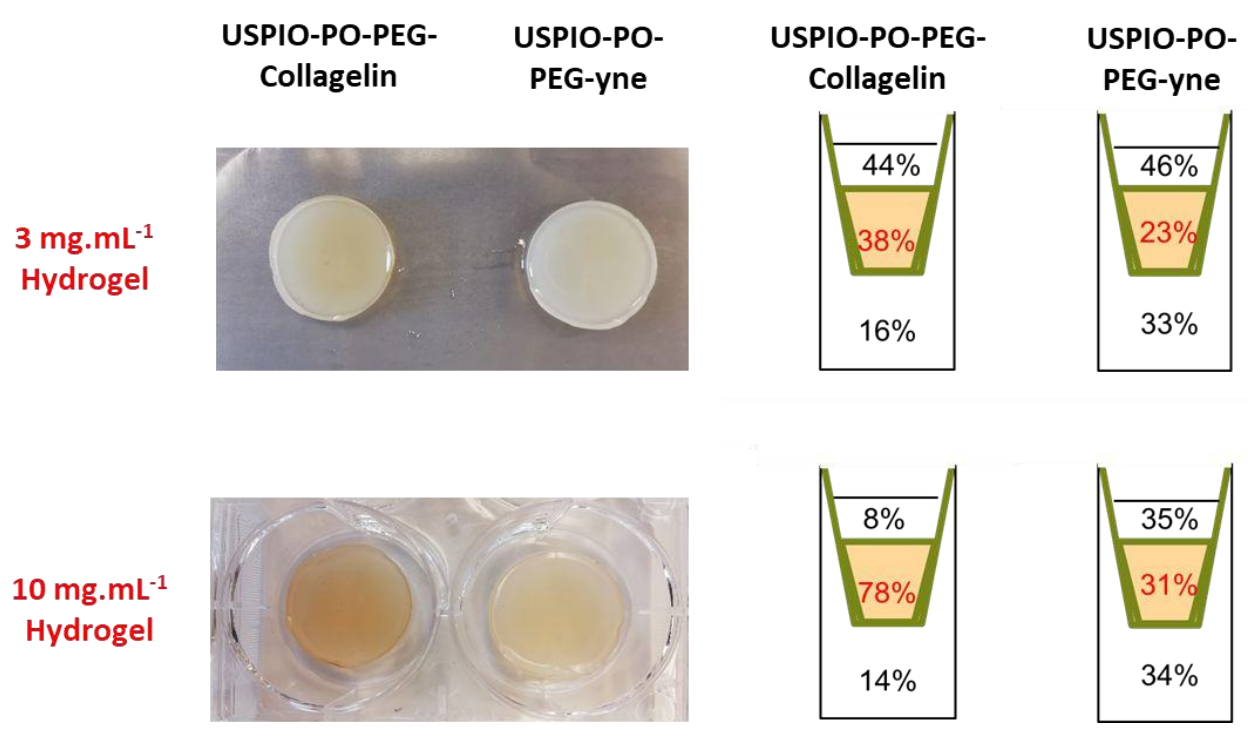

Figure 5: Distribution of USPIO-PO-PEG-Collagelin in the different compartments after 72 hours.

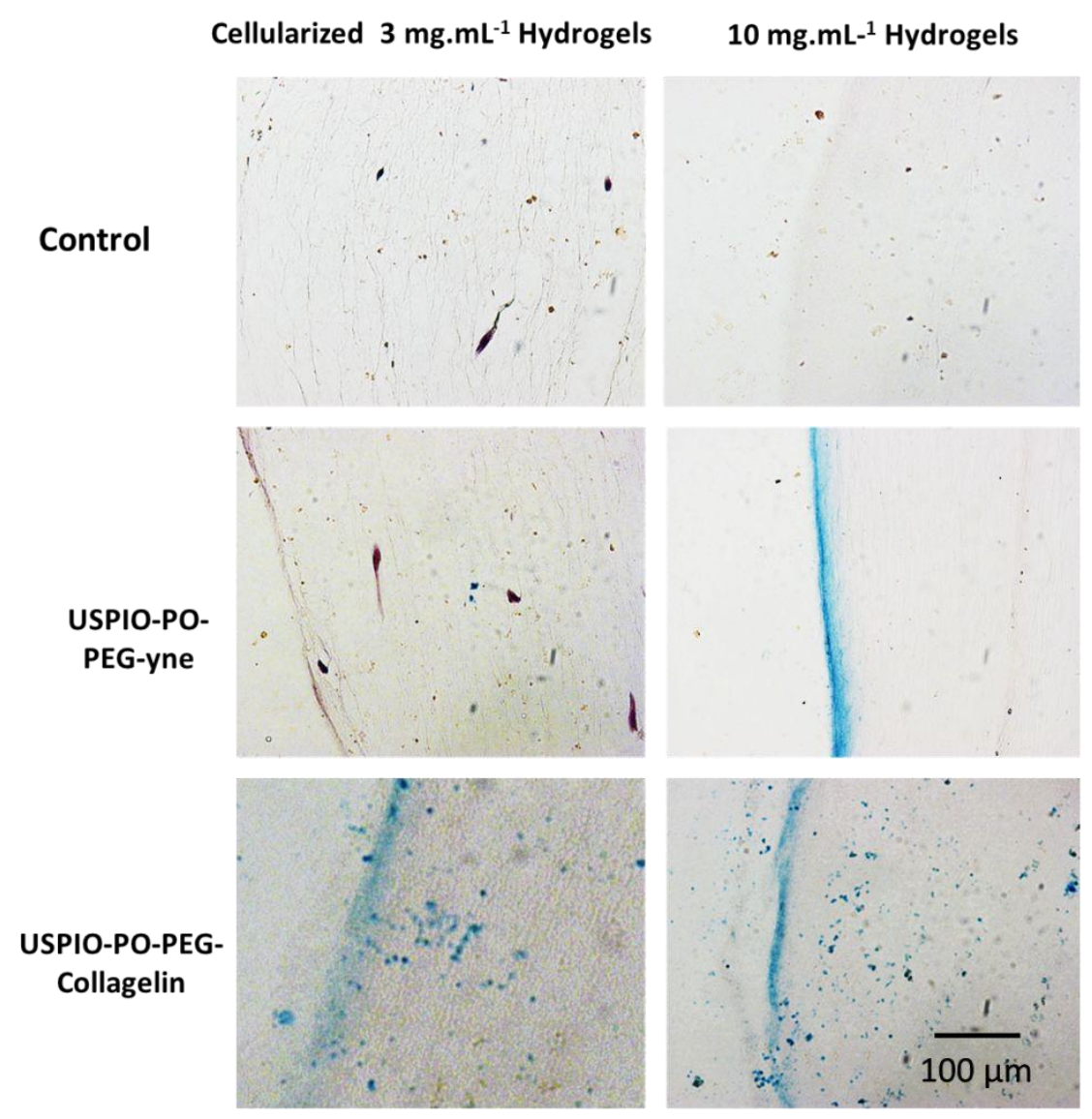

Figure 6: Iron staining on histological sections of collagen hydrogels after 72 hours of diffusion with NPs. Prussian Blue Staining. 


\subsection{Affinity of USPIO-PO-PEG-Collagelin NPs for collagen within composite collagen/alginate hydrogels.}

In order to evaluate the specificity of the USPIO-PO-PEG-Collagelin NPs for collagen in a 3D environment, collagen hydrogels (final concentration $5 \mathrm{mg} \cdot \mathrm{mL}^{-1}$ ) incorporating alginate beads were prepared and used for NP diffusion studies. The observation of gross images of the biphasic hydrogels showed that USPIO-PO-PEG-Collagelin NPs were markedly more retained than USPIO-PO-PEG-yne NPs (Figure 7A). Moreover, histological analysis on sections stained with Prussian Blue revealed the presence of blue grains within the collagen network, thereby evidencing the presence of Fe while no iron was present within the alginate beads (Figure 7C). The absence of NPs within beads was not due to a weak porosity preventing their diffusion. It has been shown that small polystyrene NPs (20 nm in diameter) diffused through alginate hydrogels ${ }^{47}$. After $72 \mathrm{~h}$ of diffusion, the amount of Fe in the various compartments were very similar to that obtained for the $3 \mathrm{mg} \cdot \mathrm{mL}^{-1}$ collagen hydrogels for both types of NPs (Figure 7B). The only noticeable difference was a decrease of Fe content in the supernatant and increase in the reservoir for USPIO-PO-PEG-yne NPS, which could reflect the change in overall network tortuosity due to the presence of large alginate beads. Most interestingly, such a difference is not observed for USPIO-PO-PEG-Collagelin NPs, highlighting again their strong interaction with collagen. 
(A)

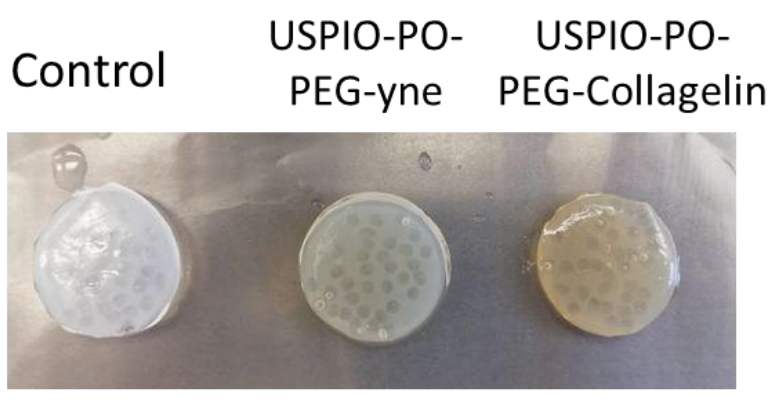

(B)

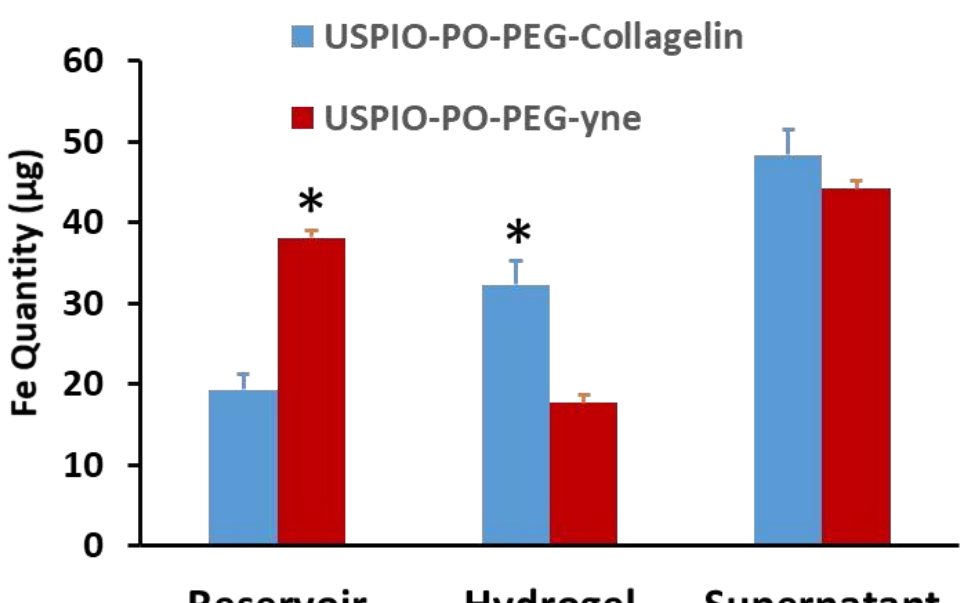

(C)

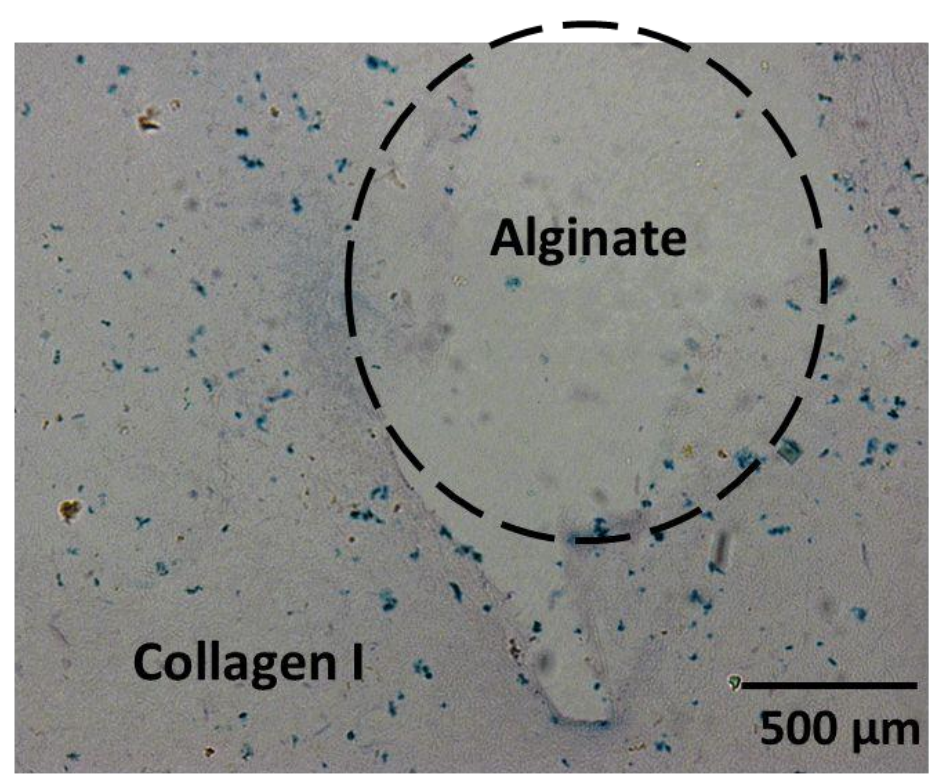

Figure 7: Diffusion, interaction and selectivity of USPIO-PO-PEG-Collagelin within biphasic alginate/collagen Hydrogels (10 mg. $\mathrm{mL}^{-1}$ alginate $+10 \mathrm{mg} \cdot \mathrm{mL}^{-1}$ Collagen) after $72 \mathrm{~h}$. (A) gross images of biphasic hydrogels. (B): diffusion profile of USPIO-PO-PEG NPs through biphasic hydrogels. (C): selective binding of USPIO-PO-PEG-Collagelin NPs on collagen compared to alginate $(*: p<0.05)$. 


\subsection{USPIO-PO-PEG-Collagelin NP: a potential MRI contrast agent for early detection of}

\section{fibrosis.}

Since USPIO NPs are negative T2 MRI contrast agents, we used local changes in T2 mapping to evaluate the diffusion profile of the NPs within collagen hydrogels after $72 \mathrm{~h}$ of NP incubation. The NPs diffusion profile is estimated from the T2 relaxation time variation (V) along an $x$-axis with and without NPs incubation. Figure 8 shows the diffusion profile measured in dense $\left(10 \mathrm{mg} \cdot \mathrm{mL}^{-1}\right)$ collagen hydrogels for the two type of NPs. Whatever the USPIO coating, the T2 relaxation time decreases along gel depth. Moreover, for USPIO-POPEG-collagelin NPs, the T2 variation remains stable from $6 \mathrm{~mm}$, at a measurable level of $30 \%$ while, USPIO-PO-PEG-yne NPs, it continues to decrease down to $20 \%$ at a $9 \mathrm{~mm}$ depth. This should reflect the fact that those USPIO-PO-PEG-collagelin NPs interacting with collagen cannot diffuse anymore (or at least at a much lower rate), insuring that they are present in a significant amount over the whole hydrogel volume. Noticeably, when these experiments were performed using $3 \mathrm{mg} \cdot \mathrm{mL}^{-1}$ collagen hydrogels, the same difference was observed between the two types of NPs but the minimum attenuation value at high depth for USPIOPO-PEG-collagelin NPs was smaller, in agreement with the lower protein concentration. (Figure ESI 6). 

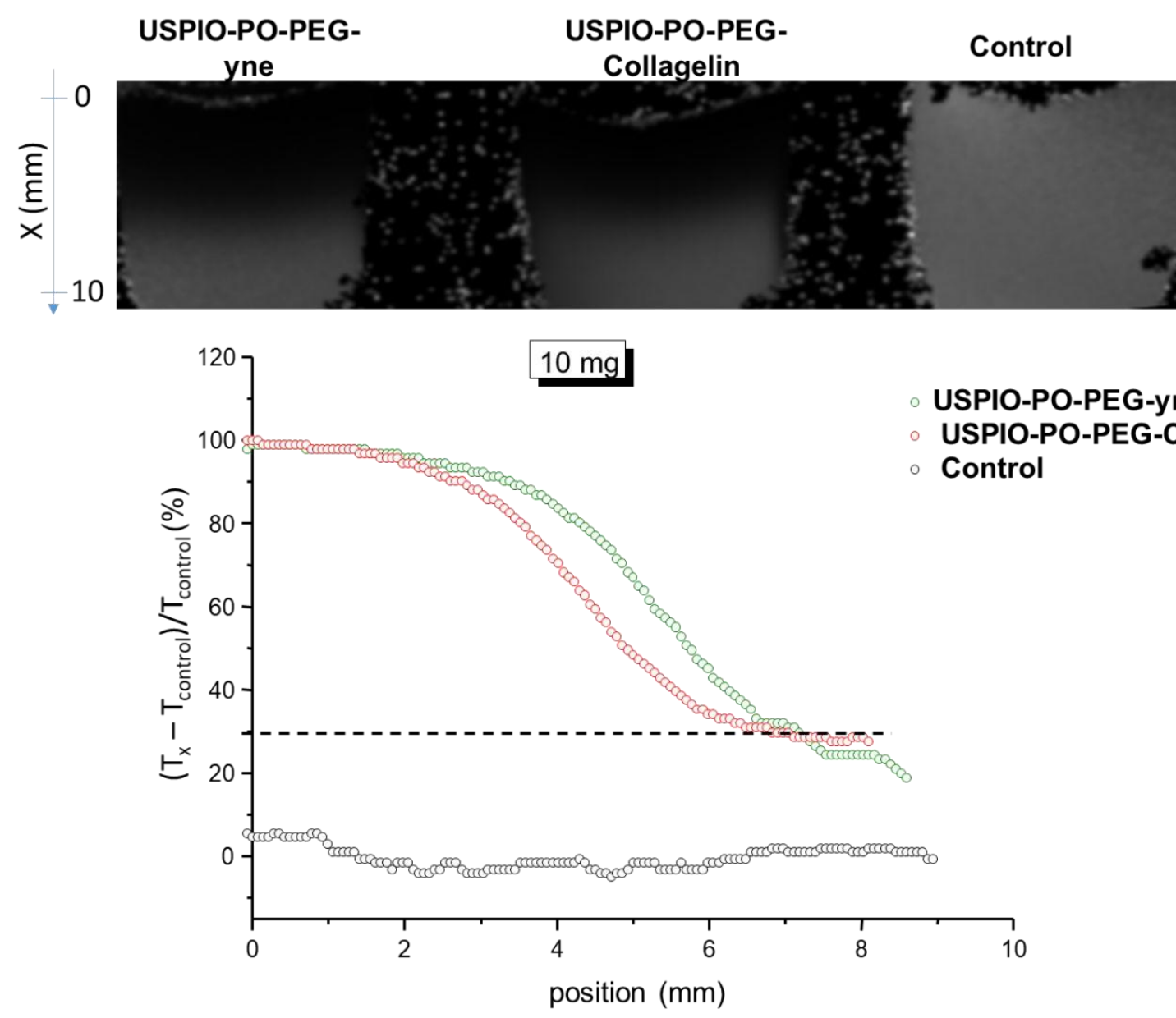

Figure 8: Evolution of the MRI attenuation signal along the hydrogel thickness (position $\mathrm{x}$ ) of $10 \mathrm{mg} \cdot \mathrm{mL}^{-1}$ collagen hydrogels (black curve) after $72 \mathrm{~h}$ incubation of USPIO-PO-PEG-yne and USPIO-PO-PEG-Collagelin NPs.

\subsection{USPIO-PO-PEG-Collagelin NPs to diagnose fibrosis}

The main drawback of the current contrast agents used in MRI to diagnose fibrosis is their inability to detect the early stage of pathology ${ }^{48}$. At the late stages characterized by irreversible anatomic alterations and an impaired tissue function, no treatment can be administrated to cure fibrosis. Hence, an early stage detection of fibrosis, when the tissue 
function is not impaired yet is of great interest. Fibrosis detection can be difficult because of the different forms of fibrotic tissues ${ }^{49}$. Nevertheless, accumulation of collagen I is the main characteristic of this pathology, and it is therefore a target of choice for imaging in a diagnosis perspective.

In this context, here-developed nanoparticles demonstrate several advantages. First, the grafting of cyclic Collagelin peptide at the surface of USPIO NPs increases its affinity for collagen. Second, USPIO NPs have better magnetic properties than Gadolinium contrast agents for MRI and are much less toxic ${ }^{19}, 50$. Finally, USPIO-PEG-Collagelin NPs properties seem better than those of free radiolabeled ${ }^{99 m} \mathrm{Tc}$ Collagelin peptide and would avoid to perform a scintigraphy, using a radioelement to detect fibrosis ${ }^{13}$. Noticeably, USPIO-PEGCollagelin NPs could diffuse and accumulate to high amount ( $80 \%$ of the initial population) within collagen hydrogels concentrated at $10 \mathrm{mg} \cdot \mathrm{mL}^{-1}$. Therefore, they would be adapted to detect the in vivo accumulation of collagen I at a very early stage when the deposition of fibrotic ECM is not large.

\section{Conclusion}

In this study, we showed that USPIO-PO-PEG NPs coupled with a collagen-binding peptide, Collagelin, specifically target type collagen and allows for its MRI detection at relatively low concentrations that mimic early stage of fibrosis. As the accumulation scales with collagen concentration, the signal attenuation in MRI could monitor the progress of the fibrotic disease. In addition, USPIO-PEG-PO-Collagelin NPs are not toxic and do not accumulate within cells such as macrophage and fibroblasts, permitting their utilization in medicine. Hence, USPIO-PEG-Collagelin NPs appears as promising effective nanotracers for MRI 
molecular imaging. Thanks to their ability to be surface functionalized with proteins, peptides or antibodies, USPIO-PO-PEG-Collagelin NPs could be also used for the delivery of growth factors at the location of fibrosis, thereby acting like a theranostic system.

\section{Conflict of Interest}

The authors declare no conflict of interest.

Acknowledgments: We warmly thank "Conseil Régional d'lle de France" program C'Nano Idf, DIM NANO K to have supported our project Nanodefi (Grant Number: DIM Nano-K 2016 NanoDFI) . The authors would like to acknowledge CNanoMat platform of the University Paris Nord for the use of UV-vis and Zetasizer Nano ZS, FRIM Imaging platform at Université Paris 7 (CHU X Bichat) for the MRI 7T measurements and member of France Life Imaging (ANR-11-INBS-0006) and IDEX Imagerie Du Vivant networks, and ICP-MS. We also thank the ImagoSeine core facility of the Institut Jacques Monod, member of IBiSA and FranceBiolmaging (ANR-10-INBS-04) infrastructures, particularly Dr. Rémi le Borgne for sample preparation and assistance with TEM imaging. 


\section{References}

1. S. Hinderer and K. Schenke-Layland, Adv Drug Deliv Rev, 2019, 146, 77-82.

2. $\quad$ B. E. Van Beers, J. L. Daire and P. Garteiser, J Hepatol, 2015, 62, 690-700.

3. $\quad$ M. Pinzani, Dig Dis, 2015, 33, 492-497.

4. D. C. Rockey, P. D. Bell and J. A. Hill, N Engl J Med, 2015, 372, 1138-1149.

5. M. Disertori, M. Mase and F. Ravelli, Trends Cardiovasc Med, 2017, 27, 363-372.

6. R. Martos, J. Baugh, M. Ledwidge, C. O'Loughlin, C. Conlon, A. Patle, S. C. Donnelly and K. McDonald, Circulation, 2007, 115, 888-895.

7. M. P. Graham-Brown, A. S. Patel, D. J. Stensel, D. S. March, A. M. Marsh, J. McAdam, G. P. McCann and J. O. Burton, Biomed Res Int, 2017, 2017, 5453606.

8. E. A. Akam, E. Abston, N. J. Rotile, H. R. Slattery, I. Y. Zhou, M. Lanuti and P. Caravan, Chemical Science, 2020, 11, 224-231.

9. H. Wahyudi, A. A. Reynolds, Y. Li, S. C. Owen and S. M. Yu, Journal of Controlled Release, 2016, 240, 323-331.

10. P. Caravan, Y. Yang, R. Zachariah, A. Schmitt, M. Mino-Kenudson, H. H. Chen, D. E. Sosnovik, G. Dai, B. C. Fuchs and M. Lanuti, Am J Respir Cell Mol Biol, 2013, 49, $1120-1126$.

11. B. C. Fuchs, H. Wang, Y. Yang, L. Wei, M. Polasek, D. T. Schuhle, G. Y. Lauwers, A. Parkar, A. J. Sinskey, K. K. Tanabe and P. Caravan, J Hepatol, 2013, 59, 992-998.

12. M. Jandrot-Perrus, J. Muzard, P. Billiald, G. D. Le, L. Sarda and A. Meulemans, Archives of cardiovascular diseases, 2009, 102, S17-S18.

13. J. Muzard, L. Sarda-Mantel, S. Loyau, A. Meulemans, L. Louedec, C. BantsimbaMalanda, F. Hervatin, J. Marchal-Somme, J. B. Michel, D. Le Guludec, P. Billiald and M. Jandrot-Perrus, PLoS One, 2009, 4, e5585.

14. U. Chilakamarthi, J. Kandhadi, S. Gunda, A. R. Thatipalli, M. Kumar Jerald, G. Lingamallu, R. C. Reddy, A. Chaudhuri and G. Pande, Exp Cell Res, 2014, 327, 91101.

15. I. Velikyan, U. Rosenstrom, S. Estrada, I. Ljungvall, J. Haggstrom, O. Eriksson and G. Antoni, Nucl Med Biol, 2014, 41, 728-736.

16. V. Hubert, C. Dumot, E. Ong, C. Amaz, E. Canet-Soulas, F. Chauveau and M. Wiart, Sci Rep, 2019, 9, 10046.

17. S. Richard, V. Eder, G. Caputo, C. Journe, P. Ou, J. Bolley, L. Louedec, E. Guenin, L. Motte, N. Pinna and Y. Lalatonne, Nanomedicine-Uk, 2016, 11, 2769-2779.

18. S. Richard, A. Saric, M. Boucher, C. Slomianny, F. Geffroy, S. Meriaux, Y. Lalatonne, P. X. Petit and L. Motte, Acs Chem Biol, 2016, 11, 2812-2819.

19. A. P. Khandhar, G. J. Wilson, M. G. Kaul, J. Salamon, C. Jung and K. M. Krishnan, J Biomed Mater Res A, 2018, 106, 2440-2447.

20. X. Ma, S. Wang, L. Hu, S. Feng, Z. Wu, S. Liu, S. Duan, Z. Chen, C. Zhou and X. Zhao, Contrast Media Mol Imaging, 2019, 2019, 3687537.

21. J. Yin, D. Yao, G. Yin, Z. Huang and X. Pu, ACS Appl Mater Interfaces, 2019, 11, 41038-41050.

22. J. Bolley, Y. Lalatonne, O. Haddad, D. Letourneur, M. Soussan, J. Perard-Viret and L. Motte, Nanoscale, 2013, 5, 11478-11489.

23. N. Erathodiyil and J. Y. Ying, Acc Chem Res, 2011, 44, 925-935.

24. S. Richard, M. Boucher, Y. Lalatonne, S. Mériaux and L. Motte, Biochimica et Biophysica Acta (BBA) - General Subjects, DOI: http://dx.doi.org/10.1016/j.bbagen.2016.12.020. 
25. S. Richard, M. Boucher, a. saric, a. herbet, y. lalatonne, P. Petit, S. Meriaux, d. boquet and L. Motte, Journal of Materials Chemistry B, 2017, DOI: 10.1039/C6TB03080G.

26. C. Sanchez, D. El Hajj Diab, V. Connord, P. Clerc, E. Meunier, B. Pipy, B. PayrÃ $\odot$, R. P. Tan, M. Gougeon, J. Carrey, V. r. Gigoux and D. Fourmy, ACS Nano, 2014, 8, 1350-1363.

27. L. Lartigue, D. Alloyeau, J. Kolosnjaj-Tabi, Y. Javed, P. Guardia, A. Riedinger, C. Péchoux, T. Pellegrino, C. Wilhelm and F. Gazeau, ACS Nano, 2013, 7, 3939-3952.

28. M. Levy, N. Luciani, D. Alloyeau, D. Elgrabli, V. Deveaux, C. Pechoux, S. Chat, G. Wang, N. Vats, F. o. Gendron, C. c. Factor, S. Lotersztajn, A. Luciani, C. Wilhelm and F. Gazeau, Biomaterials, 2011, 32, 3988-3999.

29. F. Mazuel, A. Espinosa, N. Luciani, M. Reffay, R. Le Borgne, L. Motte, K. Desboeufs, A. Michel, T. Pellegrino, Y. Lalatonne and C. Wilhelm, Acs Nano, 2016, 10, 7627-7638.

30. G. Maheshwari, G. Brown, D. A. Lauffenburger, A. Wells and L. G. Griffith, J Cell Sci, 2000, 113 ( Pt 10), 1677-1686.

31. C. de Montferrand, Y. Lalatonne, D. Bonnin, N. Lievre, M. Lecouvey, P. Monod, V. Russier and L. Motte, Small, 2012, 8, 1945-1956.

32. C. Helary, A. Abed, G. Mosser, L. Louedec, D. Letourneur, T. Coradin, M. M. Giraud-Guille and A. Meddahi-Pelle, Biomater Sci, 2015, 3, 373-382.

33. C. Helary, A. Foucault-Bertaud, G. Godeau, B. Coulomb and M. M. Guille, Biomaterials, 2005, 26, 1533-1543.

34. I. Bergman and R. Loxley, Clin Chim Acta, 1970, 27, 347-349.

35. C. Helary, I. Bataille, A. Abed, C. Illoul, A. Anglo, L. Louedec, D. Letourneur, A. Meddahi-Pellé and M. M. Giraud-Guille, Biomaterials, 2010, 31, 481-490.

36. C. Dubreil, O. Sainte Catherine, Y. Lalatonne, C. Journe, P. Ou, P. van Endert and L. Motte, Small, 2018, 14, e1802053.

37. L. Qi, A. Sehgal, J. C. Castaing, J. P. Chapel, J. Fresnais, J. F. Berret and F. Cousin, ACS Nano, 2008, 2, 879-888.

38. P. Demay-Drouhard, E. Nehlig, J. Hardouin, L. Motte and E. Guénin, Chem Eur J, 2013, 19, 8388-8392.

39. R. Sun, J. Dittrich, M. Le-Huu, M. M. Mueller, J. Bedke, J. Kartenbeck, W. D. Lehmann, R. Krueger, M. Bock, R. Huss, C. Seliger, H. J. Grone, B. Misselwitz, W. Semmler and F. Kiessling, Invest Radiol, 2005, 40, 504-513.

40. O. Lunov, T. Syrovets, B. Buchele, X. Jiang, C. Rocker, K. Tron, G. U. Nienhaus, P. Walther, V. Mailander, K. Landfester and T. Simmet, Biomaterials, 2010, 31, 50635071.

41. A. Plan Sangnier, A. B. Van de Walle, A. Curcio, R. Le Borgne, L. Motte, Y. Lalatonne and C. Wilhelm, Nanoscale, 2019, 11, 16488-16498.

42. J. Gutenwik, B. Nilsson and A. Axelsson, J Chromatogr A, 2004, 1048, 161-172.

43. S. Quignard, C. Helary, M. Boissiere, J.-M. Fullana, P.-Y. Lagree and T. Coradin, Biomater Sci, 2014, 2, 484-492.

44. R. Rattan, S. Bhattacharjee, H. Zong, C. Swain, M. A. Siddiqui, S. H. Visovatti, Y. Kanthi, S. Desai, D. J. Pinsky and S. N. Goonewardena, Bioorg Med Chem, 2017, 25, 4487-4496.

45. B. Yameen, W. I. Choi, C. Vilos, A. Swami, J. Shi and O. C. Farokhzad, J Control Release, 2014, 190, 485-499.

46. S. Quignard, G. Mosser, M. Boissière and T. Coradin, Biomaterials, 2012, 33, 44314442.

47. J. M. Rodriguez-Suarez, C. S. Butler, A. Gershenson and B. L. T. Lau, Environ Sci Technol, 2020, 54, 5159-5166. 
48. M. Baues, A. Dasgupta, J. Ehling, J. Prakash, P. Boor, F. Tacke, F. Kiessling and T. Lammers, Adv Drug Deliv Rev, 2017, 121, 9-26.

49. S. de Jong, T. A. van Veen, H. V. van Rijen and J. M. de Bakker, J Cardiovasc Pharmacol, 2011, 57, 630-638.

50. M. Rogosnitzky and S. Branch, Biometals, 2016, 29, 365-376. 

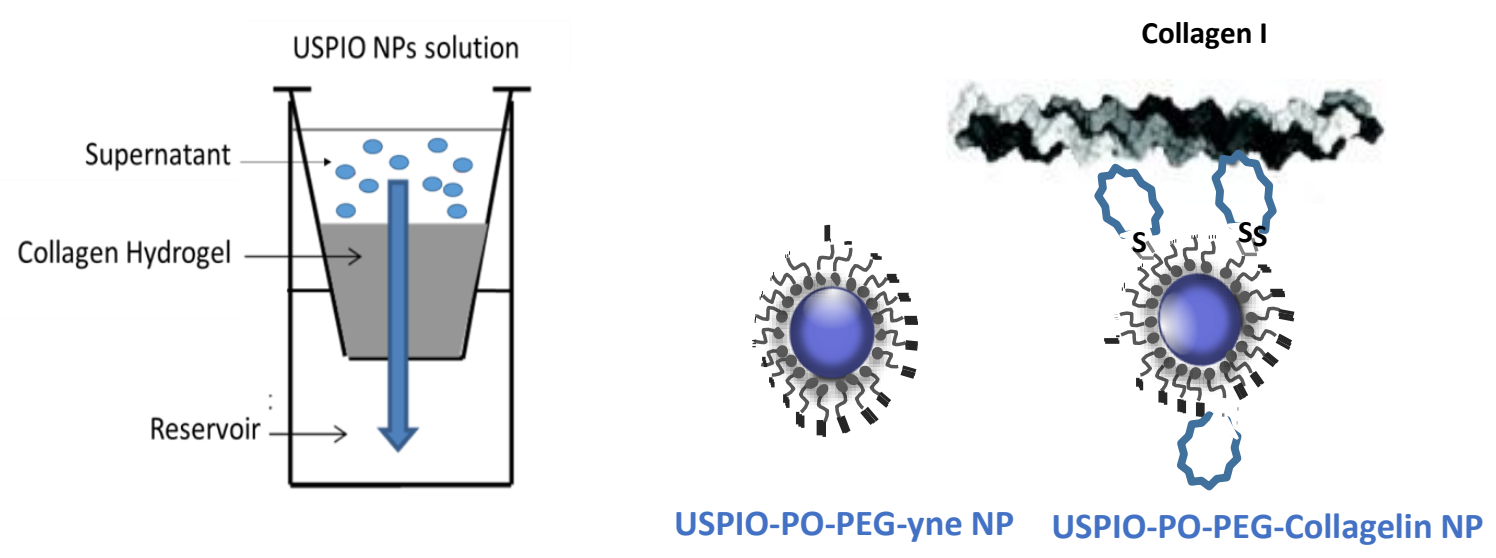

USPIO-PO-PEG-yne NP USPIO-PO-PEG-Collagelin NP

$10 \mathrm{mg} \cdot \mathrm{mL}^{-1}$
Collagen Hydrogel

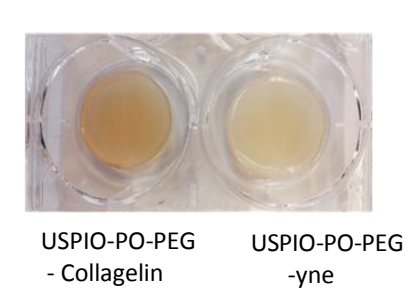

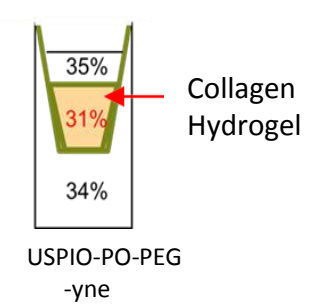

USPIO-PO-PEG-Collagelin nanoparticles specifically target type I collagen and allow fibrosis detection at an early stage by Magnetic Resonance Imaging. 